\title{
MODÈLES ENTIERS DES COURBES HYPERELLIPTIQUES SUR UN CORPS DE VALUATION DISCRÈTE
}

\author{
QING LIU
}

\begin{abstract}
Let $C$ be a hyperelliptic curve of genus $g \geq 1$ over a discrete valuation field $K$. In this article we study the models of $C$ over the ring of integers $\mathcal{O}_{K}$ of $K$. To each Weierstrass model (that is a projective model arising from a hyperelliptic equation of $C$ with integral coefficients), one can associate a (valuation of) discriminant. Then we give a criterion for a Weierstrass model to have minimal discriminant. We show also that in the most cases, the minimal regular model of $C$ over $\mathcal{O}_{K}$ dominates every minimal Weierstrass model. Some classical facts concerning Weierstrass models over $\mathcal{O}_{K}$ of elliptic curves are generalized to hyperelliptic curves, and some others are proved in this new setting.
\end{abstract}

Soit $K$ un corps de valuation discrète, d'anneau de valuation $\mathcal{O}_{K}$. Soit $C$ une courbe hyperelliptique de genre $g \geq 1$ sur $K$ (nous entendons par-là que $C$ est propre lisse, et qu'il existe un morphisme $C \rightarrow \mathbb{P}_{K}^{1}$ de degré 2 ). On étudie dans ce travail les modèles de $C$ sur $\mathcal{O}_{K}$, c'est-à-dire des schémas normaux propres et plats sur $\mathcal{O}_{K}$, à fibres génériques isomorphes à $C$.

Plus précisément, on s'intéresse aux modèles de Weierstrass de $C$ sur $\mathcal{O}_{K}$. Ce sont des modèles provenant des équations hyperelliptiques de $C$ à coefficients dans $\mathcal{O}_{K}$ (déf. 6). On étudie aussi la relation entre les modèles de Weierstrass et le modèle régulier minimal de $C$ sur $\mathcal{O}_{K}$.

Pour tout modèle de Weierstrass de $C$ sur $\mathcal{O}_{K}$, il y a une notion évidente du discriminant (§4.3). Le discriminant minimal de $C$ est un invariant particulièrement important dans l'étude de l'arithmétique de $C$, comme en témoignent les travaux sur les courbes elliptiques. D'ailleurs, ce travail généralise certains résultats bien connus sur les modèles de Weierstrass entiers des courbes elliptiques.

Dans les trois premiers paragraphes, on rappelle la notion du discriminant et on définit les équations minimales et le discriminant minimal de $C$. On montre de façon tout à fait élémentaire que, sur un anneau principal, $C$ admet une équation minimale globale (prop. 2).

La notion du discriminant est liée au choix d'une involution hyperelliptique $\sigma$ sur $C$. Si $g \geq 2, \sigma$ est unique. Par contre, si $g=1$, cela n'est plus vrai. Dans $\S 1$, on étudie l'ensemble des classes de conjugaison (par les automorphismes intérieurs de $\left.\operatorname{Aut}_{K}(C)\right)$ des involutions hyperelliptiques de $C$, et on donne une estimation de

Received by the editors August 22, 1995.

1991 Mathematics Subject Classification. Primary 11G20, 14H25; Secondary 14G20.

Key words and phrases. Courbe hyperelliptique, modèle de Weierstrass, discriminant. 
son cardinal (prop. 1). On verra plus tard que le discriminant minimal de $C$ est indépendant du choix de $\sigma$ si $C(K) \neq \emptyset$ ou si la caractéristique du corps résiduel $k$ est différente de 2 (remarque 21 ).

Dans les paragraphes 4 à 6 , on introduit la notion du modèle de Weierstrass de $C$ sur $\mathcal{O}_{K}$. Pour tout point fermé $p_{0}$ d'un tel modèle $W$, on associe une multiplicité $\lambda\left(p_{0}\right) \in \mathbb{N}$ (déf. 10) qui mesure la singularité de $p_{0}$ dans $W$. Cette multiplicité est en général différente de la multiplicité de l'anneau local de $W$ en $p_{0}$ et du nombre de Milnor en $p_{0}$. Quelques résultats techniques préliminaires sur ces multiplicités sont démontrés.

Cette notion de multiplicité permet d'énoncer un critère pour qu'un modèle de Weierstrass $W$ soit (de discriminant) minimal ( $(77$, cor. 2). Dans le même paragraphe, on étudie le comportement du discriminant minimal de $C$ vis-à-vis d'un changement de base $\mathcal{O}_{L} / \mathcal{O}_{K}$ non-ramifiée (prop. 4).

À partir du paragraphe 8 , on s'intéresse au modèle régulier minimal $X$ de $C$ sur $\mathcal{O}_{K}$. Pour tout modèle de Weierstrass minimal $W$ de $C$ sur $\mathcal{O}_{K}$, on donne un critère pour que $X$ domine $W$, autrement dit que la désingularisation minimale de $W$ soit isomorphe à $X$ (prop. 5 et 6 ). On en déduit (cor. 4) que $C$ n'admet qu'un nombre fini de modèles de Weierstrass minimaux (à involution hyperelliptique fixée si $g(C)=1)$.

Par une étude élémentaire du résultant, on déduit des relations entre le discriminant d'un modèle de Weierstrass $W$ et les multiplicités des points fermés de $W$ (prop. 7). Cela conduit aussi à une majoration des longueurs des chaînes de type $\mathrm{A}_{n}$ ou $\mathrm{D}_{n}$ dans la fibre spéciale $X_{k}$ de $X$ (lemme 13 et cor. 8). Une notion de facteurs locaux du discriminant permet d'améliorer ces majorations (cor. 7), mais malheureusement nous ne savons construire ces facteurs locaux que si $\operatorname{car}(k) \neq 2$.

Enfin, on applique les résultats précédents au cas classique et bien connu des courbes elliptiques $(\S 10)$. On montre que le modèle régulier minimal $X$ domine le modèle de Weierstrass minimal de la courbe elliptique (remarque 20). Une preuve de ce fait sans recours à l'algorithme de Tate ne semblait pas être connue dans la littérature. Par l'étude de l'action de l'involution hyperelliptique sur $X_{k}$, on simplifie légèrement la preuve de l'algorithme de Tate ([Sil], IV.9) pour les types $\mathrm{II}^{*}$, III* et $\mathrm{IV}^{*}$. Nous décrivons aussi tout l'algorithme de Tate en utilisant les multiplicités $\lambda\left(p_{0}\right)$, ce qui permet un traitement plus systématique et qui s'applique à un cadre plus général que les courbes de genre 1 .

La notion du discriminant minimal adopté dans ce travail est une notion "naïve". Des considérations plus élaborées en utilisant le faisceau dualisant relatif sur le modèle régulier minimal sont plus adaptées dans d'autres contextes (voir [Uen], [Sai], [Liu2] ou plus récemment [Kau]). Cependant on peut déterminer explicitement la différence entre ces deux sortes de discriminants, au moins dans le cas du genre $g=2([\operatorname{Liu} 2], \S 6)$.

La motivation de départ de ce travail était de construire un algorithme "à la Tate" pour la détermination de la réduction des courbes de genre 2. Mais faute de temps d'une part, et faute de place d'autre part, cette question n'a pas été abordée ici excepté dans le cadre général des courbes hyperelliptiques. Cependant un tel algorithme existe pour $\operatorname{car}(k) \neq 2([\operatorname{Liu} 1])$. Son implémentation sur $\mathbb{Z}[1 / 2]$ sous PARI (disponible par ftp sur le site : megrez.math.u-bordeaux.fr /pub/liu) a été réalisée avec l'aide d'Henri Cohen. Je le remercie ici pour son dévouement et sa patience. Je remercie également Pascal Létard et Emmanuel Tollis qui m'ont fait bénéficier de leur connaissance de PARI. 


\section{Equations hyperelliptiques}

Dans ce paragraphe, $K$ est un corps quelconque. On ne considère pas de valuation sur $K$.

\subsection{Involutions et équations hyperelliptiques.}

Lemme 1. Soit $A$ un corps ou un anneau principal. Soit $B$ la clôture intégrale de $A[x]$ dans une extension séparable $F$ de degré 2 sur $\operatorname{Fr}(A[x])$. Alors on a les propriétés suivantes :

(a) Le $A[x]$-module $B$ est libre et admet une base de la forme $\{1, y\}$.

(b) Supposons que $F$ est le corps de fonctions d'une courbe projective lisse de genre $g$ sur $\operatorname{Fr}(A)$. On peut choisir la base $\{1, y\}$ de sorte que si $y^{2}+Q(x) y=P(x)$, alors $\operatorname{deg} Q(x) \leq g+1$ et $\operatorname{deg} P(x) \leq 2 g+2$.

Preuve. (a) Voir [Liu2], lemme 1.

(b) Soit $K=\operatorname{Fr}(A)$. Il existe une base $\{1, z\}$ de $B \otimes K$ sur $K[x]$ avec $z^{2}+$ $R(x) z=S(x), \operatorname{deg} R(x) \leq g+1$ et $\operatorname{deg} S(x) \leq 2 g+2$. Quitte à multiplier $z$ par un élément de $K^{*}$, on peut supposer que $R, S \in A[x]$. Donc il existe $a \in A \backslash\{0\}$, $T(x) \in A[x]$ tels que $z=a y+T(x)$. Si $a \in A^{*}$, alors $\{1, z\}$ est une base qui répond à la question. Supposons donc $a \notin A^{*}$. Soit $t \in A$ un élément irréductible divisant $a$. On peut écrire $T(x)=T_{0}(x)+t T_{1}(x)$ avec $T_{i}(x) \in \mathcal{O}_{K}[x]$ et aucun des coefficients de $T_{0}(x)$ n'appartient à $t A$. Comme $T_{0}(x)^{2}+Q(x) T_{0}(x)-P(x)$ est un multiple de $t$, on a $\operatorname{deg} T_{0}(x) \leq g+1$. On peut donc remplacer $z$ par $z_{1}:=\left(z-T_{0}(x)\right) / t=(a / t) y+T_{1}(x)$. Si $a / t \in A^{*}$, c'est terminé, sinon on continue comme ci-dessus. Cela s'arrête au bout d'un nombre fini de fois.

Définition 1. On appellera base standard toute base $\{1, y\}$ satisfaisant les conditions du lemme 1 (b) ci-dessus.

Soit $C$ une courbe hyperelliptique de genre $g \geq 2$ sur $K$. Soit $\sigma$ l'unique involution hyperelliptique sur $C$. Alors le corps $K(C)^{\langle\sigma\rangle}$ (des éléments invariants par $\sigma$ ) est l'unique sous- $K$-extension transcendante pure d'indice 2 de $K(C)$. Autrement dit, le morphisme $C \rightarrow \mathbb{P}_{K}^{1}$ de degré 2 est unique à automorphismes de $\mathbb{P}_{K}^{1}$ près.

Si $g=1$, on fixera dans toute la suite une involution hyperelliptique $\sigma$ de $K(C)$, c'est-à-dire une involution de $K(C)$ telle que $K(C)^{\langle\sigma\rangle}$ soit une extension transcendante pure sur $K$.

Une équation (hyperelliptique) de $C$ est la donnée d'un générateur $x$ de $K(C)^{\langle\sigma\rangle}$ et d'un élément $y \in K(C)$ tel que $\{1, y\}$ soit une base standard de la clôture intégrale de $K[x]$ dans $K(C)$. On présente cette équation sous la forme

$$
(\mathcal{E}): \quad y^{2}+Q(x) y=P(x)
$$

où les polynômes $Q(x), P(x) \in K[x]$ sont les opposés respectivement de la trace de $y$ et de la norme de $y$ sur $K[x]$. Par hypothèse, $\operatorname{deg} Q \leq g+1$ et $\operatorname{deg} P \leq 2 g+2$. Lorsque $\operatorname{car}(K) \neq 2$, on choisit souvent $y$ avec $Q(x)=0$.

Étant donnée une équation hyperelliptique $(\mathcal{E})$ comme ci-dessus, on peut retrouver la courbe $C$ comme recouvrement de deux ouverts affines

$$
C=\operatorname{Spec} K[x, y] \cup \operatorname{Spec} K[v, z]
$$

où les variables vérifient les relations $y^{2}+Q(x) y=P(x), z^{2}+Q_{1}(v) z=P_{1}(v)$ où $Q_{1}(v)=v^{g+1} Q(1 / v)$ et $P_{1}(x)=v^{2 g+2} P(1 / v)$. Les deux ouverts se recollent par les relations $x v=1, z x^{g+1}=y$. 
1.2 Les involutions hyperelliptiques d'une courbe de genre 1.

Supposons dans cette section que $C$ est de genre 1. On étudie ici les classes de conjugaison des involutions hyperelliptiques sur $C$.

Notons $E$ la jacobienne de $C$ et $\operatorname{Pic}_{K}^{r}(C) \subset \mathrm{H}^{1}\left(C, \mathcal{O}_{C}^{*}\right)$ l'ensemble des diviseurs de degré $r$ (définis sur $K$ ) de $C$ modulo équivalence linéaire. Si $D$ est un diviseur sur $C$, on note $[D]$ sa classe d'équivalence linéaire. On a une injection canonique $\operatorname{Pic}_{K}^{0}(C) \rightarrow E(K)$ ([Mil1], Remark 1.6). Il est important ici de distinguer la courbe $C$ et la variété abélienne $E$ même lorsque $C$ admet des points rationnels.

Soit $\sigma$ une involution hyperelliptique sur $C$, soit $\pi: C \rightarrow C /\langle\sigma\rangle$ le morphisme canonique. Alors pour tout point rationnel $\infty$ de $C /\langle\sigma\rangle, D_{\sigma}:=\pi^{*} \infty$ est un diviseur de degré 2 sur $C$, invariant par $\sigma$. L'image $\left[D_{\sigma}\right]$ de $D_{\sigma}$ dans $\operatorname{Pic}_{K}^{2}(C)$ ne dépend que de $\sigma$. Inversement, à tout diviseur effectif $D$ de degré 2 sur $C$, on peut associer une involution hyperelliptique qui provient du revêtement $C \rightarrow \mathbb{P}(L(D))=\mathbb{P}_{K}^{1}$, car $\operatorname{dim}_{K} L(D)=2$. Cela établit une bijection entre l'ensemble des involutions hyperelliptiques de $C$ et $\operatorname{Pic}_{K}^{2}(C)$.

Pour tout $f \in \operatorname{Aut}_{K}(C), f \sigma f^{-1}$ est une involution hyperelliptique associée à $f\left(D_{\sigma}\right)$. Donc l'ensemble des classes de conjugaison des involutions hyperelliptiques est en bijection avec les orbites de $\operatorname{Pic}_{K}^{2}(C)$ sous l'action de $\operatorname{Aut}_{K}(C)$. Notons cet ensemble $\operatorname{Aut}_{K}(C) \backslash \operatorname{Pic}_{K}^{2}(C)$.

Proposition 1. Soient $C$ une courbe hyperelliptique de genre 1, E sa jacobienne et $\mathcal{H}$ l'ensemble des classes de conjugaison des involutions hyperelliptiques de $C$. On a les inégalités sur les cardinaux:

$$
\left|\operatorname{Pic}_{K}^{0}(C) / 2 E(K)\right| \geq|\mathcal{H}| \geq \frac{2}{\left|\operatorname{Aut}_{K}(E)\right|}\left|\operatorname{Pic}_{K}^{0}(C) / 2 E(K)\right|
$$

(les membres peuvent être infinis).

Preuve. Montrons d'abord qu'il existe une suite exacte de groupes

$$
0 \rightarrow E(K) \rightarrow \operatorname{Aut}_{K}(C) \stackrel{\iota}{\rightarrow} \operatorname{Aut}_{K}(E) .
$$

Le groupe $\operatorname{Aut}_{K}(C)$ opère naturellement $\operatorname{sur} \operatorname{Pic}_{K}^{0}(C)$, cela induit par fonctorialité un homomorphisme de groupes $\iota: \operatorname{Aut}_{K}(C) \rightarrow \operatorname{Aut}_{K}(E)$. D'autre part, tout point rationnel $a \in E(K)$ induit un élément $t_{a} \in \operatorname{Aut}_{K}(C)$ défini de la façon suivante : sur la clôture algébrique $\bar{K}$ de $K$, pour tout point $\bar{p} \in C(\bar{K}), t_{a}(\bar{p})$ est l'unique point de $C(\bar{K})$ tel que $\left[t_{a}(\bar{p})\right]=[\bar{p}]+a$. Si $p \in C$, alors $t_{a}(p)$ est un point qui vérifie $\left[t_{a}(p)\right]=[p]+(\operatorname{deg} p) a$ (notons que $(\operatorname{deg} p) a \in \operatorname{Pic}_{K}^{0}(C)$ ). Cela induit un homomorphisme de groupes $E(K) \rightarrow \operatorname{Aut}_{K}(C)$ qui est injectif car il est injectif sur $\bar{K}$. De plus, il est clair que son image est contenue dans $\operatorname{Ker}(\iota)$. Si $C(K) \neq \emptyset$, la suite ci-dessus est clairement exacte. Dans le cas général, on considère la suite exacte sur une clôture séparable $K^{s}$ et on prend les invariants par $\operatorname{Gal}\left(K^{s} / K\right)$.

Montrons maintenant les inégalités. On a vu plus haut que l'ensemble $\mathcal{H}$ est en bijection avec l'espace des orbites $\operatorname{Aut}_{K}(C) \backslash \operatorname{Pic}_{K}^{2}(C)$. Soient $X:=E(K) \backslash \operatorname{Pic}_{K}^{2}(C)$ et $G=\operatorname{Im} \iota$, alors $\mathcal{H} \simeq G \backslash X$. Comme l'image par $\iota$ de toute involution hyperelliptique est l'automorphisme $(-1)_{E}$, celui-ci opère trivialement sur $X$. Donc on a

$$
|X| \geq|\mathcal{H}| \geq \frac{2}{\left|\operatorname{Aut}_{K}(E)\right|}|X| .
$$

Fixons un diviseur $D_{0}$ de degré 2 sur $C$. Alors $a \mapsto\left[D_{0}\right]+a$ définit une application bijective $\operatorname{Pic}_{K}^{0}(C) / 2 E(K) \rightarrow X$. Ce qui prouve le résultat. 
Remarque 1. D'après cette proposition, l'ensemble $\mathcal{H}$ des classes de conjugaison des involutions hyperelliptiques sur $C$ peut être infini en général, même dans le cas $C(K) \neq \emptyset$. Cependant, $E(K) / 2 E(K)$ (et donc $\mathcal{H}$ ) est fini si $K$ est un corps de nombres (Mordell-Weil) ou un corps local (utiliser le groupe formel de $E$ ).

\section{LE DISCRIMINANT D'UNE ÉQUATION HYPERELLIPTIQUE}

Si $z^{2}+T(u) z=S(u)$ est une autre équation de $C$, alors $K(u)=K(x)$. Donc il existe

$$
\left(\begin{array}{ll}
a & b \\
c & d
\end{array}\right) \in \mathrm{GL}_{2}(K), \quad e \in K^{*}, \quad H(u) \in K[u]
$$

tels que

$$
x=\frac{a u+b}{c u+d}, \quad y=\frac{e z+H(u)}{(c u+d)^{g+1}} .
$$

Le discriminant d'une équation hyperelliptique. Considérons deux polynômes à coefficients génériques

$$
\begin{gathered}
\mathcal{Q}(x)=u_{g+1} x^{g+1}+u_{g} x^{g}+\cdots+u_{0} \in \mathbb{Z}\left[u_{0}, \ldots, u_{g+1}\right][x], \\
\mathcal{P}(x)=v_{2 g+2} x^{2 g+2}+v_{2 g+1} x^{2 g+1}+\cdots+v_{0} \in \mathbb{Z}\left[v_{0}, \ldots, v_{2 g+2}\right][x] .
\end{gathered}
$$

Soit

$$
\Delta\left(\left(u_{i}\right)_{i},\left(v_{j}\right)_{j}\right):=2^{-4(g+1)} \operatorname{disc}\left(4 \mathcal{P}(x)+\mathcal{Q}(x)^{2}\right) .
$$

On peut montrer comme dans [Loc], Theorem 1.7, que $\Delta\left(\left(u_{i}\right)_{i},\left(v_{j}\right)_{j}\right) \in \mathbb{Z}\left[u_{i}, v_{j}\right]_{i, j}$. Soit

$$
(\mathcal{E}): \quad y^{2}+\left(b_{g+1} x^{g+1}+b_{g} x^{g}+\cdots+b_{0}\right) y=a_{2 g+2} x^{2 g+2}+a_{2 g+1} x^{2 g+1}+\cdots+a_{0}
$$

une équation de $C$. Alors on pose

$$
\Delta(\mathcal{E}):=\Delta\left(\left(b_{i}\right)_{i},\left(a_{j}\right)_{j}\right) .
$$

Le fait que $C$ soit lisse sur $K$ se traduit par $\Delta(\mathcal{E}) \neq 0$.

Supposons $\operatorname{car}(K) \neq 2$. Soit $(\mathcal{E}): y^{2}+Q(x) y=P(x)$ une équation hyperelliptique de $C$, avec $R(X):=4 P(x)+Q(x)^{2}$ de coefficient dominant $c$. Alors

$$
\Delta(\mathcal{E})=\left\{\begin{array}{lll}
2^{-4(g+1)} \operatorname{disc}(R(x)) & \text { si } & \operatorname{deg} R(x)=2 g+2, \\
2^{-4(g+1)} c^{2} \operatorname{disc}(R(x)) & \text { si } & \operatorname{deg} R(x)=2 g+1 .
\end{array}\right.
$$

Soient $y^{2}+Q(x) y=P(x), z^{2}+T(u) z=S(u)$ deux équations de $C$ sur $K$. Soit $\Delta$ le discriminant de la première équation et $\Delta^{\prime}$ celui de la seconde. Posons $n=2 g+2$. On sait que le discriminant est un invariant projectif de degré $2 n-2$ et d'indice $n(n-1)$. Donc avec les notations du début du paragraphe, on a la relation suivante :

$$
\begin{aligned}
\Delta^{\prime} & =\Delta e^{-4(n-1)}(a d-b c)^{n(n-1)} \\
& =\Delta e^{-4(2 g+1)}(a d-b c)^{2(g+1)(2 g+1)} .
\end{aligned}
$$




\section{EQUATIONS ENTIÈRES ET ÉQUATIONS MINIMALES}

Fixons quelques hypothèses et notations pour la suite. Soient

$K$ un corps de valuation discrète, d'uniformisante $t$, $\mathcal{O}_{K}$ son anneau de valuation,

$\nu: K \rightarrow \mathbb{Z} \cup\{\infty\}$ la valuation normalisée de $K$,

$k$ son corps résiduel. On supposera $k$ parfait pour simplifier.

On note $\widetilde{a}$ l'image de $a$ dans $k$ pour tout $a \in \mathcal{O}_{K}$.

La valuation $\nu$ s'étend en une valuation, notée encore par $\nu$, sur l'anneau de polynômes $\mathcal{O}_{K}[x]$ en posant $\nu\left(\sum_{i} a_{i} x^{i}\right)=\min _{i}\left\{\nu\left(a_{i}\right)\right\}$. Pour tout élément $P(x)=$ $\sum_{i} a_{i} x^{i} \in \mathcal{O}_{K}[x]$, on note $\widetilde{P}(x)=\sum_{i} \widetilde{a}_{i} x^{i}$ son image dans $k[x]$.

Lemme 2. Soient $P(x), Q(x) \in \mathcal{O}_{K}[x], B=\mathcal{O}_{K}[x, y] /\left(y^{2}+Q(x) y-P(x)\right)$. Supposons que $B \otimes K$ est normal. Les propriétés suivantes sont vraies.

(a) Si $B \otimes k$ est réduite, alors $B$ est normal.

(b) L'algèbre $B \otimes k$ n'est pas réduite si et seulement si $4 \widetilde{P}(x)+\widetilde{Q}(x)^{2}=0$ et si $-\widetilde{P}(x)$ est un carré dans $k[x]$.

(c) Supposons $B \otimes k$ non réduite. Alors $B$ est normal si et seulement s'il existe $R(x) \in \mathcal{O}_{K}[x]$ tel que $\nu\left(P(x)+Q(x) R(x)-R(x)^{2}\right)=1$.

(d) Si $\operatorname{car}(k) \neq 2$, alors $B$ est normal si et seulement si $\nu\left(4 P(x)+Q(x)^{2}\right) \leq 1$.

Preuve. (a) C'est élémentaire.

(b) Supposons que le nilradical $I$ de $B \otimes k$ soit non trivial. Comme $B \otimes k$ est libre de rang 2 sur $k[x], I$ est libre de rang 1 . Il est aisé de voir alors que l'homomorphisme canonique $k[x] \rightarrow(B \otimes k) / I$ est un isomorphisme. Donc il existe $\widetilde{R}(x) \in k[x]$ tel que $z:=y+\widetilde{R}(x) \in I$. On a $I=z k[x]=z(B \otimes k)$, donc $z^{2}=0$. Ce qui équivaut à $4 \widetilde{P}(x)+\widetilde{Q}(x)^{2}=0$ et que $-\widetilde{P}(x)$ est un carré dans $k[x]$. La réciproque est immédiate.

(c) Soit $R(x) \in \mathcal{O}_{K}[x]$ tel que $\widetilde{P}(x)+\widetilde{R}(x)^{2}=0$. En choisissant convenablement le signe de $R(x)$, on a $\widetilde{Q}(x)=2 \widetilde{R}(x)$. Quitte à remplacer $y$ par $y+R(x)$, on peut supposer que $\widetilde{Q}(x)=\widetilde{P}(x)=0$. Si $\nu(P) \geq 2$, alors $B$ n'est pas normal car $y / t$ est entier sur $B$. Inversement, supposons $\nu(P)=1$. Soit $\{1, w\}$ une base sur $\mathcal{O}_{K}[x]$ de la clôture intégrale de $B$ (voir lemme 1). On a $y=a w+T(x)$ avec $a \in \mathcal{O}_{K}$ et $T(x) \in \mathcal{O}_{K}[x]$. En reportant cette relation dans l'équation de $y$ sur $\mathcal{O}_{K}[x]$, on voit que $a \in \mathcal{O}_{K}^{*}$, donc $w \in B$ et $B$ est normal.

(d) On peut supposer $Q=0$. L'assertion découle alors des résultats précédents.

Remarque 2. La preuve du lemme ci-dessus contient aussi le procédé de normalisation de $B=\mathcal{O}_{K}[x, y] /\left(y^{2}+Q(x) y-P(x)\right)$.

Si $\operatorname{car}(k) \neq 2$, on se ramène d'abord à $Q(x)=0$ en remplaçant $y$ par $y+Q / 2$. Soit $c=\nu(P)$, alors la normalisation de $B$ s'obtient en remplaçant $y$ par $y / t^{[c / 2]}$.

Supposons maintenant $\operatorname{car}(k)=2$. On peut supposer $\widetilde{Q}(x)=0$ et qu'il existe $R(x) \in \mathcal{O}_{K}[x]$ tel que $\widetilde{P}(x)+\widetilde{R}(x)^{2}=0$ (sinon $B$ est normal). Cela revient à dire que $\widetilde{Q}(x)=0$ et $\widetilde{P}(x) \in k\left[x^{2}\right]$. Si $P(x)=\sum a_{i} x^{i}$, on peut prendre $R(x)=\sum b_{i} x^{i}$ où $b_{i} \in \mathcal{O}_{K}$ est tel que $\widetilde{b}_{i}^{2}+\widetilde{a}_{2 i}=0$. Si $\nu\left(P+R Q-R^{2}\right)=1$, on a fini. Sinon, on remplace $y$ par $(y+R) / t$, et on recommence. Ce procédé s'arrête au bout d'un nombre fini de fois car la valuation du discriminant diminue strictement chaque fois. 
Remarque 3. Soient $K(x)=K(C)^{\langle\sigma\rangle}$ et $B$ la clôture intégrale de $\mathcal{O}_{K}[x]$ dans $K(C)$. On voit dans la preuve du lemme ci-dessus que si $B \otimes k$ n'est pas réduite, alors $B$ admet une base standard $\{1, y\}$ telle que $\widetilde{Q}(x)=\widetilde{P}(x)=0$.

Définition 2. Une équation entière de $C$ est une équation hyperelliptique

$$
y^{2}+Q(x) y=P(x)
$$

de $C$ telle que $\{1, y\}$ soit une base standard de la clôture intégrale de $\mathcal{O}_{K}[x]$ dans $K(C)$.

Notons que cette propriété est plus forte que de supposer simplement $Q(x), P(x)$ à coefficients dans $\mathcal{O}_{K}$.

Remarque 4. Soit $(\mathcal{E}): z^{2}+Q_{1}(x) z=P_{1}(x)$ une équation de $C$ à coefficients dans $\mathcal{O}_{K}$, soit $\left(\mathcal{E}_{1}\right): y^{2}+Q(x) y=P(x)$ une équation entière de $C$ avec la même variable $x$. Alors $\nu\left(\Delta\left(\mathcal{E}_{1}\right)\right) \leq \nu(\Delta(\mathcal{E}))$. Cela résulte du fait que $z \in y \mathcal{O}_{K}+\mathcal{O}_{K}[x]$.

Définition 3. Une équation entière $(\mathcal{E})$ de $C$ sera dite minimale si $\nu(\Delta(\mathcal{E}))$ est minimal parmi toutes les équations entières de $C$. L'entier $\nu(\Delta(\mathcal{E}))$ est alors appelé discriminant minimal de $C\left(\operatorname{sur} \mathcal{O}_{K}\right)$, on le notera $\nu(C)$.

Lorsque $g=1$, ces notions sont relatives au choix d'une involution hyperelliptique $\sigma$. Elles sont équivalentes pour des involutions hyperelliptiques de la même classe de conjugaison (au sens de $\$ 1.2$ ). Voir aussi la remarque 21.

On dit que $C$ a bonne réduction si $\nu(C)=0$. Cela équivaut à dire que $C$ est la fibre générique d'un schémas propre lisse sur $\mathcal{O}_{K}$.

Remarque 5. Une équation minimale existe toujours, mais elle n'est pas unique en général, même modulo l'action de $\mathrm{PGL}_{2}\left(\mathcal{O}_{K}\right)$. On peut considérer l'exemple qui suit.

Supposons $\operatorname{car}(k) \neq 2,3$. Soit $C$ la courbe de genre 2 définie par une équation $y^{2}=\left(x^{3}+a\right)\left(x^{3}+t^{6}\right), a \in \mathcal{O}_{K}^{*}$. C'est une équation minimale, dont la valuation du discriminant vaut 12 . Le changement de variables $x=t^{2} / u, y=t^{3} z / u^{3}$ donne $z^{2}=\left(u^{3}+1\right)\left(a u^{3}+t^{6}\right)$. C'est une autre équation minimale. En fait ces deux équations définissent deux modèles de Weierstrass non isomorphes (§4).

Lemme 3. Soit $A$ un sous-anneau de $\mathcal{O}_{K}$ contenant t et tel que $A / t A \simeq \mathcal{O}_{K} / t \mathcal{O}_{K}$. Soit $y^{2}+Q(x) y=P(x)$ une équation de $C$ à coefficients dans $\operatorname{Fr}(A)$. Alors il existe un changement de variables $x=a u+b, y=c z+R(u)$ avec $a, c \in t^{\mathbb{Z}}, b \in A[1 / t]$ et $R(u) \in A[1 / t][u]$ tel que la nouvelle équation $z^{2}+Q_{1}(u) z=P_{1}(u)$ soit minimale.

Preuve. (1) Montrons d'abord le résultat avec $a, b, c \in K$ et $R(u) \in K[u]$. Il existe un changement de variables $x=(\alpha u+\beta) /(\gamma u+\delta), y=(\epsilon z+H(u)) /(\gamma u+\delta)^{g+1}$, qui donne une équation en $u, z$ qui est minimale. Si $\gamma=0$, il n'y a rien à démontrer. Sinon, soit $\eta=-\delta / \gamma$. On compose avec le changement de variables $u=(\eta w-1) / w$, $z=v / w^{g+1}$ ou $u=w /\left(\eta^{-1} w+1\right), z=v /\left(\eta^{-1} w+1\right)^{g+1}$ suivant que $\nu(\eta) \geq 0$ ou $<0$. Cela donne alors le résultat. En effet, il est clair que l'équation en $w, v$ est à coefficients dans $\mathcal{O}_{K}$ et a le même discriminant que l'équation en $u, z$.

(2) Montrons le lemme. Soit $x=a_{1} u_{1}+b_{1}, y=c_{1} z_{1}+R_{1}\left(u_{1}\right)$ un changement de variables dans $K$ qui donne une équation minimale. Comme $A[1 / t]$ est dense dans $K$, on peut écrire $b_{1}=b+b_{2}$, avec $b \in A[1 / t]$ et $\nu\left(b_{2}\right)>N:=\nu\left(a_{1}\right)$. Posons $u=\left(t^{-N} a_{1}\right) u_{1}+t^{-N} b_{2}$. Alors $x=a u+b$ avec $a=t^{N}$. On a $R_{1}\left(u_{1}\right)=R_{2}(u) \in K[u]$. En approximant de la même façon $R_{2}(u)$ par un élément $R(u) \in A[1 / t][u]$, on peut 
écrire $y=c z+R(u)$ avec $c=t^{\nu\left(c_{1}\right)}$. Il est clair que l'équation en $u, z$ ainsi obtenue est à coefficients dans $A$, de discriminant minimal.

Proposition 2. Soit $A$ un anneau de Dedekind. Soient $C$ une courbe hyperelliptique sur $\operatorname{Fr}(A)$ et $y^{2}+Q(x) y=P(x)$ une équation de $C$ sur $\operatorname{Fr}(A)$, de discriminant $\Delta$. Supposons que tout idéal maximal $\mathfrak{p}$ de $A$ tel que $\nu_{\mathfrak{p}}(\Delta) \neq 0$ soit principal. Alors il existe une équation hyperelliptique de $C$ à coefficients dans $A$ qui soit minimal dans $A_{\mathfrak{p}}$ pour tout idéal maximal $\mathfrak{p}$ de $A$.

Preuve. Soient $\mathfrak{p}_{1}, \ldots, \mathfrak{p}_{n}$ les idéaux maximaux de $A$ tels que $\nu_{\mathfrak{p}_{i}}(\Delta) \neq 0$. En appliquant successivement un changement de variables comme dans le lemme 3 à $\mathcal{O}_{K}=A_{\mathfrak{p}_{1}}, \ldots, A_{\mathfrak{p}_{n}}$, on obtient le résultat. En effet un tel changement de variables en une place $\mathfrak{p}_{i}$ ne modifie pas la nature de l'équation en toute place distincte de $\mathfrak{p}_{i}$.

Corollaire 1. Soit $A$ un anneau principal. Soit $C$ une courbe hyperelliptique sur $\operatorname{Fr}(A)$ ayant bonne réduction en toute place finie de $A$. Alors $C$ admet une équation $(\mathcal{E})$ à coefficients dans $A$ et de discriminant $\Delta(\mathcal{E})$ inversible.

Remarque 6. Sur un anneau de Dedekind $A$, on peut définir deux idéaux de $A$ associés à $C$ ayant la propriété qu'ils sont tous deux principaux si et seulement s'il existe une équation minimale globale sur $A$.

\section{Modèles de Weierstrass}

\subsection{Modèles propres normaux.}

Définition 4. Soit $E$ une courbe propre lisse et géométriquement connexe sur $K$. Un modèle de $E$ sur $\mathcal{O}_{K}$ est un schéma normal $X$, propre et plat sur $\mathcal{O}_{K}$, muni d'un isomorphisme $\phi_{X}: X_{K} \rightarrow E$ sur $K$. Deux modèles $X, Y$ sont dits isomorphes s'il existe un isomorphisme de $\mathcal{O}_{K}$-schéma $X \rightarrow Y$ compatible avec l'isomorphisme $\phi_{Y}^{-1} \circ \phi_{X}$. Dans la suite, l'isomorphisme $\phi_{X}$ sera généralement omis dans les notations.

Le fait suivant (une conséquence immédiate du "Zariski's Main Theorem") est important pour les modèles normaux, il sera souvent utilisé implicitement.

Soient $X, Y$ deux modèles de $C$ sur $\mathcal{O}_{K}$. On suppose qu'il existe

une application rationnelle $f: X \rightarrow Y$ telle que $f$ et $f^{-1}$ soient

définies en tous les points de codimension 1, alors $f$ est un isomorphisme.

Autrement dit, un modèle $X$ est complètement déterminé par les anneaux locaux de $X$ aux points générique de la fibre spéciale $X_{k}$.

4.2 Modèles de $\mathbb{P}_{K}^{1}$.

Soit $x$ un générateur de $K\left(\mathbb{P}_{K}^{1}\right)$ sur $K$. Notons $\mathbb{P}_{x}^{1}$ le $\mathcal{O}_{K}$-schéma

$$
\mathbb{P}_{x}^{1}:=\operatorname{Spec} \mathcal{O}_{K}[x] \cup \operatorname{Spec} \mathcal{O}_{K}[1 / x] .
$$

Alors $\mathbb{P}_{x}^{1} \simeq \mathbb{P}_{\mathcal{O}_{K}}^{1}$ et c'est un modèle de $\mathbb{P}_{K}^{1}$ sur $\mathcal{O}_{K}$. Inversement, tout modèle de $\mathbb{P}_{K}^{1}$ lisse sur $\mathcal{O}_{K}$ s'obtient de cette façon. Deux modèles $\mathbb{P}_{x}^{1}, \mathbb{P}_{u}^{1}$ sont isomorphes si et seulement s'il existe

$$
\left(\begin{array}{ll}
a & b \\
c & d
\end{array}\right) \in \mathrm{GL}_{2}\left(\mathcal{O}_{K}\right)
$$

tel que $x=(a u+b) /(c u+d)$. 
Plus généralement, soient $Z_{1}=\mathbb{P}_{x}^{1}, Z_{2}=\mathbb{P}_{u}^{1}$ deux modèles lisses de $\mathbb{P}_{K}^{1}$. Quitte à remplacer $x$ et $u$ par des conjugués (sous l'action de $\operatorname{PGL}_{2}\left(\mathcal{O}_{K}\right)$ ) convenables, on peut supposer que $x=t^{d} u$. L'entier $\mathrm{d}\left(Z_{1}, Z_{2}\right):=|d|$ ne dépend que des modèles $Z_{1}, Z_{2}$.

Définition 5. Soient $Z_{1}, Z_{2}$ deux modèles lisses de $\mathbb{P}_{K}^{1}$. On appelle distance entre $Z_{1}, Z_{2}$ l'entier $\mathrm{d}\left(Z_{1}, Z_{2}\right)$ ci-dessus. Cela définit une métrique sur l'ensemble des classes d'isomorphismes des modèles lisses de $\mathbb{P}_{K}^{1}$ sur $\mathcal{O}_{K}$.

Si $r:=d\left(Z_{1}, Z_{2}\right)>0$, on peut écrire $Z_{1}=\mathbb{P}_{x}^{1}, Z_{2}=\mathbb{P}_{u}^{1}$ avec $x=t^{r} u$. On appellera l'intersection de $Z_{1}, Z_{2}$ et la note $Z_{1} \wedge Z_{2}$ l'ensemble des deux points $\{x=t=0\} \in Z_{1},\{1 / u=t=0\} \in Z_{2}$.

Soit $Y$ un modèle quelconque de $\mathbb{P}_{K}^{1}$, soit $\Theta$ une composante irréductible de $Y_{k}$ de multiplicité 1. Alors il existe un unique modèle lisse $Z$ et un morphisme $\phi: Y \rightarrow Z$ de modèles tel que $\phi\left(\Theta^{\prime}\right)$ soit réduit à un point pour toute composante irréductible $\Theta^{\prime}$ de $Y_{k}$ distinctes de $\Theta$, et que $\left.\phi\right|_{\Theta}: \Theta \rightarrow Z_{k}$ soit birationnel.

\subsection{Modèles de Weierstrass d'une courbe hyperelliptique.}

Définition 6. Soit $\sigma$ l'involution hyperelliptique fixe de $C$. Un modèle de Weierstrass de $C\left(\operatorname{sur} \mathcal{O}_{K}\right)$ est un modèle $W$ de $C$ sur $\mathcal{O}_{K}$ tel que $\sigma$ opère sur $W$ et que $W /\langle\sigma\rangle$ soit lisse $\operatorname{sur} \mathcal{O}_{K}$.

Soit $x$ un générateur de $K(C)^{\langle\sigma\rangle}$ sur $K$. Soit $W$ la normalisation de $\mathbb{P}_{x}^{1}$ dans $C$. Alors $W$ est un modèle de Weierstrass de $C$ sur $\mathcal{O}_{K}$. On dira que $W$ est le modèle de Weierstrass de $C$ associé à $x$. Tout modèle de Weierstrass de $C$ est obtenu de cette manière à isomorphisme près. Ainsi l'ensemble des classes d'isomorphisme des modèles de Weierstrass de $C$ s'identifie à l'ensemble des classes d'isomorphisme des modèles lisses de $C /\langle\sigma\rangle \simeq \mathbb{P}_{K}^{1}$. Enfin ce dernier s'identifie (non canoniquement) à $\mathrm{PGL}_{2}\left(\mathcal{O}_{K}\right) \backslash \mathrm{PGL}_{2}(K)$.

Soient $W_{1}, W_{2}$ deux modèles de Weierstrass $C$ associés respectivement à $x$ et $u$. Alors $W_{1}, W_{2}$ sont deux modèles isomorphes s'il et seulement si $\mathbb{P}_{x}^{1} \simeq \mathbb{P}_{u}^{1}$ en tant que modèles de $\mathbb{P}_{K}^{1}$, c'est-à-dire que $u$ est dans l'orbite de $x$ sous l'action de $\mathrm{PGL}_{2}\left(\mathcal{O}_{K}\right)$.

Définition 7. Soient $W_{1}, W_{2}$ deux modèles de Weierstrass de $C, Z_{i}=W_{i} /\langle\sigma\rangle$. On note $\mathrm{d}\left(W_{1}, W_{2}\right)$ la distance $\mathrm{d}\left(Z_{1}, Z_{2}\right)$, et $W_{1} \wedge W_{2}$ l'image réciproque de $Z_{1} \wedge Z_{2}$ dans la réunion disjointe $W_{1} \amalg W_{2}$ si $W_{1} \nsucceq W_{2}$ (voir déf. 5).

Soit $B$ la clôture intégrale de $\mathcal{O}_{K}[x]$ dans $K(C)$, soit $\{1, y\}$ une base standard de $B$ sur $\mathcal{O}_{K}[x]$ (voir $\S 1$ ). Cela correspond donc à une équation entière $(\mathcal{E})$ : $y^{2}+Q(x) y=P(x)$ de $C$. Notons $\nu\left(\Delta_{W}\right):=\nu(\Delta(\mathcal{E}))$. C'est un entier indépendant du choix de $x$. En effet, si $u$ donne le même modèle de Weierstrass, on a $x=$ $(a u+b) /(c u+d)$ comme ci-dessus, et $\left\{1, y /(c u+d)^{g+1}\right\}$ est une base de la clôture intégrale de $\mathcal{O}_{K}[u]$ dans $K(C)$.

Définition 8. Un modèle de Weierstrass minimal de $C$ ( $\operatorname{sur} \mathcal{O}_{K}$ ) est un modèle de Weierstrass de $C$ tel que $\nu\left(\Delta_{W}\right)$ soit minimal parmi tous les modèles de Weierstrass de $C \operatorname{sur} \mathcal{O}_{K}$. Notons que $W$ est lisse sur $\mathcal{O}_{K}$ si et seulement si $\nu\left(\Delta_{W}\right)=0$.

Remarque \%. Supposons que $C$ contient un point de Weierstrass $P_{0}$ rationnel sur $K$. Lockhart [Loc] a étudié le discriminant minimal de la courbe pointée $\left(C, P_{0}\right)$. 
Il s'agit du plus petit discriminant des modèles de Weierstrass $W$ de $C$ sur $\mathcal{O}_{K}$ tels que la spécialisation de $P_{0}$ dans $W_{k}$ soit lisse sur $k$ (voir déf. 14). Le discriminant minimal que nous étudions ici est naturellement (et parfois strictement) plus petit.

\section{Points singuliers D'Un modèle de Weierstrass}

Soit $W$ un modèle de Weierstrass de $C$. On s'intéresse aux points singuliers du schéma $W$. Comme $W_{K}=C$, les points singuliers de $W$ se trouvent tous dans la fibre spéciale $W_{k}$. De plus, ils sont en nombre fini car $W$ est normal et de dimension 2 .

Lemme 4. Soit $A$ un anneau local noethérien régulier, d'idéal maximal $\mathfrak{m}$. Soit $f \in \mathfrak{m}$ non nul. Alors $A / f A$ est régulier si et seulement si $f \notin \mathfrak{m}^{2}$.

Preuve. Soit $\mathfrak{m}_{f}=\mathfrak{m} / f A$ l'idéal maximal de $A / f A$. Soit $k$ le corps résiduel de $A$ (et donc de $A / f A$ ). On a une suite exacte canonique de $k$-espaces vectoriels

$$
0 \rightarrow\left(\mathfrak{m}^{2}+f A\right) / \mathfrak{m}^{2} \rightarrow \mathfrak{m} / \mathfrak{m}^{2} \rightarrow \mathfrak{m}_{f} / \mathfrak{m}_{f}^{2} \rightarrow 0
$$

Comme $\operatorname{dim} A / f A=\operatorname{dim} A-1, A / f A$ est régulier si et seulement $\operatorname{si}\left(\mathfrak{m}^{2}+f A\right) / \mathfrak{m}^{2} \neq$ 0 , c'est-à-dire que $f \notin \mathfrak{m}^{2}$.

Notations. Fixons quelques notations pour la suite. Soit $p_{0}$ un point fermé de $W_{k}$. Soit $q_{0} \in W /\langle\sigma\rangle$ l'image de $p_{0}$. Fixons $x \in K(C)^{\langle\sigma\rangle}$ tel que $W /\langle\sigma\rangle=\mathbb{P}_{x}^{1}$ (voir $\S 4$ ) et que $q_{0} \in \operatorname{Spec} \mathcal{O}_{K}[x]$. On note $\mathfrak{m}_{q_{0}}$ l'idéal maximal de $\mathcal{O}_{K}[x]$ correspondant au point $q_{0}$ et $B$ la clôture intégrale de $\mathcal{O}_{K}[x]$ dans $K(C)$. Pour toute base $\{1, y\}$ de $B$ sur $\mathcal{O}_{K}[x]$, on note $Q(x)=-\operatorname{Tr}(y), P(x)=-\operatorname{Norm}(y)$ (voir $\S 1$ ). On note aussi parfois $S_{0}(x) \in \mathcal{O}_{K}[x]$ un polynôme unitaire tel que $m_{q_{0}}=\left(t, S_{0}(x)\right)$.

Lemme 5. Avec les notations ci-dessus, on a les propriétés suivantes :

(a) Soit $\{1, y\}$ une base de B. Si le polynôme

$$
s(x):=\operatorname{pgcd}\left\{4 \widetilde{P}(x)+\widetilde{Q}(x)^{2}, \widetilde{P}^{\prime}(x)^{2}+\widetilde{Q}(x) \widetilde{Q}^{\prime}(x) \widetilde{P}^{\prime}(x)-\widetilde{P}(x) \widetilde{Q}^{\prime}(x)^{2}\right\}
$$

n'appartient pas à $m_{q_{0}}$, alors $p_{0}$ est régulier dans $W_{k}$ et dans $W$.

(b) Supposons $s(x) \in \mathfrak{m}_{q_{0}}$. Alors il existe une base standard $\{1, y\}$ de $B$ telle que $Q(x), P(x) \in \mathfrak{m}_{q_{0}}$.

(c) Sous les hypothèses de (b), on a $p_{0}$ régulier dans $W$ si et seulement si $P(x) \notin$ $\mathfrak{m}_{q_{0}}^{2}$.

Preuve. (a) On détermine les points réguliers de $\operatorname{Spec} B \otimes k$ à l'aide du critère jacobien. Comme $W_{k}$ est un diviseur de Cartier dans $W$, tout point régulier dans $W_{k}$ est régulier dans $W$.

(b) On peut supposer car $(k)=2$, le cas contraire étant trivial (il suffit de prendre $y$ avec $Q(x)=0)$. Soit $\{1, y\}$ une base standard. Soit $s_{0}(x) \in k[x]$ un polynôme unitaire qui engendre l'idéal $\mathfrak{m}_{q_{0}} \otimes k$ de $k[x]$. Par hypothèse, $s_{0}(x)$ divise $\widetilde{Q}$. Donc si $\widetilde{Q} \neq 0$, alors $\operatorname{deg} s_{0}(x) \leq \operatorname{deg} \widetilde{Q}(x) \leq g+1$. Si $\widetilde{Q}(x)=0$, alors $s_{0}(x)$ divise $\widetilde{P}^{\prime}(x), \operatorname{donc} \operatorname{deg} s_{0}(x)<\operatorname{deg} \widetilde{P}(x) / 2 \leq g+1$. Il existe donc $\widetilde{H} \in k[x]$ tel que $\widetilde{P}+\widetilde{H}^{2} \in s_{0}(x) k[x]$ (on utilise le fait que $k[x] /\left(s_{0}(x)\right)$ est un corps parfait) et que $\operatorname{deg} \widetilde{H}(x) \leq g$. On relève $\widetilde{H}$ en $H(x) \in \mathcal{O}_{K}[x]$ de même degré. Après le changement de variables $y=z-H(x)$, on peut supposer que $s_{0}(x) \mid \widetilde{Q}(x)$ et $s_{0}(x) \mid \widetilde{P}(x)$. Autrement dit, $Q(x), P(x) \in \mathfrak{m}_{q_{0}}$.

(c) Supposons maintenant que $y$ est choisi de cette manière. Soit $\mathfrak{m}_{p_{0}}$ l'idéal maximal de $B$ correspondant à $p_{0}$. Considérons l'anneau de polynômes $\mathcal{O}_{K}[x, Y]$, 
$\mathfrak{m}$ l'idéal maximal $\left(\mathfrak{m}_{q_{0}}, Y\right)$ et $f=Y^{2}+Q(x) Y-P(x) \in \mathfrak{m}$. Alors $\mathfrak{m}_{0}=\mathfrak{m} /(f)$ et $f+P(x) \in \mathfrak{m}^{2}$. Il suit du lemme 4 (appliqué à $A=\mathcal{O}_{K}[X, Y]_{\mathfrak{m}}$ ) que $p_{0}$ est un point régulier de $\operatorname{Spec} B$ si et seulement si $P(x) \notin \mathfrak{m}^{2}$, ce qui équivaut à $P(x) \notin \mathfrak{m}_{q_{0}}^{2}$.

En résumé : Supposons $W_{k}$ réduit. Les points singuliers de $\operatorname{Spec} B$ sont à chercher parmi les points au-dessus de l'ensemble des zéro $V(s(x)) \subset \operatorname{Spec} k[x]$.

Si $W_{k}$ n'est pas réduit, on se ramène d'abord à $\widetilde{P}=\widetilde{Q}=0$ (voir remarque 3 ). Soit $P_{1}(x)=P(x) / t \in \mathcal{O}_{K}[x]$. Les points singuliers de Spec $B$ sont exactement ceux qui sont au-dessus de $V\left(\widetilde{P}_{1}(x)\right)$.

\section{Multiplicités Des POINTS FERMÉS D'Un MOdÈle DE Weierstrass}

Dans tout ce paragraphe, on fixe un modèle de Weierstrass $W$ de $C$ sur $\mathcal{O}_{K}$. Pour tout point fermé $p_{0} \in W_{k}$, on choisit arbitrairement un élément $x$ tel que $W /\langle\sigma\rangle=\mathbb{P}_{x}^{1}$ et que l'image $q_{0}$ de $p_{0}$ dans $W /\langle\sigma\rangle$ appartienne à $\operatorname{Spec} \mathcal{O}_{K}[x]$. On note $B$ la clôture intégrale de $\mathcal{O}_{K}[x]$ dans $K(C)$. On utilisera librement d'autres notations introduites au $\S 5$. On va définir des entiers $\lambda_{r}\left(p_{0}\right)$ relatif au point $p_{0}$ qui mesure d'une certaine manière la singularité de $W$ en $p_{0}$.

\subsection{Multiplicité dans la fibre spéciale.}

Pour tout $h(x) \in k[x]$, posons $\delta\left(p_{0}, h(x)\right):=$ l'exposant de $\mathfrak{m}_{q_{0}}$ dans $h(x)$. Par convention, $\delta\left(p_{0}, 0\right)=+\infty$. Soit $\{1, y\}$ une base (non nécessairement standard) de $B$ sur $\mathcal{O}_{K}[x]$, Si $W_{k}$ est réduit, on pose

$$
\delta\left(p_{0}, y\right)=\min \left\{2 \delta\left(p_{0}, \widetilde{Q}(x)\right), \delta\left(p_{0}, \widetilde{P}(x)\right)\right\}
$$

Dans le cas contraire, on prend $y$ tel que $Q(x), P(x) \in t \mathcal{O}_{K}[x]$ (voir la remarque $3)$, et on pose

$$
\delta\left(p_{0}, y\right)=\delta\left(p_{0}, \widetilde{P}_{1}(x)\right)
$$

où $P_{1}(x)=P(x) / t \in \mathcal{O}_{K}[x]$. Il est à noter que $\delta\left(p_{0}, y\right)$ ne dépend pas du choix de $y$ dans ce cas-là.

Définition 9. Soit $p_{0} \in W_{k}$. Si $W_{k}$ est réduit, on note $\delta\left(p_{0}\right)$ le maximum des $\delta\left(p_{0}, y\right)$ pour les bases (non nécessairement standard) $\{1, y\}$ de $B$ sur $\mathcal{O}_{K}[x]$. Sinon, on pose $\delta\left(p_{0}\right)=\delta\left(p_{0}, y\right)$ pour une base standard $\{1, y\}$ telle que $\widetilde{Q}(x)=\widetilde{P}(x)=0$.

Supposons $\operatorname{car}(k) \neq 2$, alors $\delta\left(p_{0}\right)=\delta\left(p_{0}, y\right)$ si $Q(x)=0$.

Lemme 6. Soient $W$ un modèle de Weierstrass de $C$ sur $\mathcal{O}_{K}, p_{0} \in W_{k}$ un point fermé.

(a) Supposons $W_{k}$ réduit. On a l'équivalence : $p_{0}$ lisse sur $k \Longleftrightarrow \delta\left(p_{0}\right) \leq 1$.

(b) Supposons $W_{k}$ réduit. Soit $\pi: \widetilde{W_{k}} \rightarrow W_{k}$ la normalisation de $W_{k}$. Si $\delta\left(p_{0}\right) \geq$ 2 est pair, alors $\left(\widetilde{W}_{k}\right)_{p_{0}} \rightarrow$ Spec $k\left(p_{0}\right)$ est séparable de degré 2 . Si $\delta\left(p_{0}\right)$ est impair, $\pi$ est un homéomorphisme topologique au-dessus de $p_{0}$. Enfin

$$
\operatorname{dim}_{k}\left(\pi_{*} \mathcal{O}_{\widetilde{W_{k}}}\right)_{p_{0}} / \mathcal{O}_{W_{k}, p_{0}}=\left[\delta\left(p_{0}\right) / 2\right] .
$$

(c) Si $W_{k}$ n'est pas réduit, ou si $\operatorname{car}(k) \neq 2$, alors

$$
\sum_{p \in W_{k}}[k(p): k] \delta(p)=2 g+2 .
$$


(d) Si $W_{k} \rightarrow(W /\langle\sigma\rangle)_{k}$ est séparable, alors

$$
\sum_{p \in W_{k}}[k(p): k] \delta(p) \leq 2 g+2 .
$$

(e) Dans le cas autre que (c) et (d), on a

$$
\sum_{p \in W_{k}}[k(p): k](\delta(p)-1)=2 g .
$$

(f) Soit $p_{1} \neq p_{0}$ un point de $W_{k}$, alors $\delta\left(p_{0}\right)+\delta\left(p_{1}\right) \leq 2 g+2$.

Preuve. Soient $s_{0}(x)$ un générateur de $\mathfrak{m}_{q_{0}}, y$ tel que $\delta\left(p_{0}, y\right)=\delta\left(p_{0}\right)$, et $l=$ $\left[\delta\left(p_{0}\right) / 2\right]$ la partie entière de $\delta\left(p_{0}\right) / 2$.

(a) Si $\delta\left(p_{0}\right) \geq 2$, alors $y / s_{0}(x)^{l}$ est entier sur $B \otimes k$ mais $y / s_{0}(x)^{l} \notin B \otimes k$. Il suit que $p_{0}$ n'est pas normal dans $W_{k}$, il n'est donc pas lisse sur $k$. Si $\delta\left(p_{0}\right) \leq 1$, $W_{k}$ est clairement lisse en $p_{0}$.

(b) Considérons $D=k\left[x, y / s_{0}(x)^{l}\right]$. Il résulte de (a) que $\operatorname{Spec} D \rightarrow \operatorname{Spec}(B \otimes k)$ est la normalisation aux points $p_{0}$ et $\sigma\left(p_{0}\right)$. De plus, pour tout point $p_{0}^{\prime} \in \operatorname{Spec} D$ au-dessus de $p_{0}$, sa multiplicité $\delta\left(p_{0}^{\prime}\right)$ calculée dans $D$ vérifie $\delta\left(p_{0}^{\prime}\right)=\delta\left(p_{0}\right)-2 l$. On en déduit aisément le résultat.

(c)-(d) Supposons d'abord que $W_{k}$ n'est pas réduit. Soit $\{1, y\}$ une base standard telle que $\widetilde{Q}(x)=\widetilde{P}(x)=0$. Alors pour tout point $p \in \operatorname{Spec}(B \otimes k)$, on a $\delta(p, y)=$ $\delta\left(p, \widetilde{P}_{1}(x)\right)$ où $P_{1}(x)=P(x) / t \in \mathcal{O}_{K}[x]$. Donc

$$
\sum_{p \in \operatorname{Spec}(B \otimes k)}[k(p): k] \delta(p)=\operatorname{deg} \widetilde{P}_{1}(x) .
$$

Or au-dessus de $x=\infty$, cette somme vaut $(2 g+2)-\operatorname{deg} \widetilde{P}_{1}(x)$, d'où l'égalité souhaitée.

Supposons maintenant $W_{k}$ réduit. Si $\operatorname{car}(k) \neq 2$, on montre qu'il y a égalité exactement comme ci-dessus en prenant $Q=0$. Supposons désormais $\operatorname{car}(k)=2$. $\operatorname{Si} \widetilde{Q}(x) \neq 0\left(W_{k} \rightarrow(W /\langle\sigma\rangle)_{k}\right.$ est alors séparable $)$, alors $\delta(p)=\delta(p, y)=2 \delta(p, \widetilde{Q}(x))$ pour tout $p \in \operatorname{Spec} B \otimes k$. On conclut comme ci-dessus.

(e) On a $\operatorname{car}(k)=2, W_{k}$ réduit et $\widetilde{Q}(x)=0$ pour toute base $\{1, y\}$ de $B$. Pour tout $p \in \operatorname{Spec}(B \otimes k)$, on a $\delta(p)-1=\delta\left(p_{0}, \widetilde{P}^{\prime}(x)\right)$ où $P^{\prime}(x)$ est la dérivée de $P(x)$. L'égalité s'obtient comme dans le cas $W_{k}$ non réduit.

(f) résulte immédiatement de (c)-(e).

Remarque 8. Si $W_{k}$ est réduit et si $p_{0} \in W_{k}$ est singulier, alors $p_{0}$ est une singularité de type $\mathrm{A}_{n}$ avec $n=\delta\left(p_{0}\right)-1$.

Calcul effectif de $\delta\left(p_{0}\right)$.

Si $W_{k}$ n'est pas réduit, $\delta\left(p_{0}\right)$ se calcule avec une base standard quelconque $\{1, y\}$ de $B$ telle que $\widetilde{Q}(x)=\widetilde{P}(x)=0$. Si $W_{k}$ est réduit mais $\operatorname{car}(k) \neq 2$, alors $\delta\left(p_{0}\right)=$ $\delta\left(p_{0}, 4 \widetilde{P}(x)+\widetilde{Q}(x)^{2}\right)$ pour une base $\{1, y\}$ quelconque de $B$.

Supposons maintenant $W_{k}$ réduit et $\operatorname{car}(k)=2$. Si $2 \delta\left(p_{0}, \widetilde{Q}(x)\right) \leq \delta\left(p_{0}, \widetilde{P}(x)\right)$ ou si $\delta\left(p_{0}, \widetilde{P}(x)\right)$ est impair, alors $\delta\left(p_{0}\right)=\delta\left(p_{0}, y\right)$. Sinon, soit $s_{0}(x)$ un générateur de $\mathfrak{m}_{q_{0}}$, et écrivons $\widetilde{P}(x)=\sum_{i \geq 2 n} c_{i}(x) s_{0}(x)^{i}$ dans la base $s_{0}(x)$-adique. Soit $\delta\left(p_{0}, \widetilde{P}(x)\right)=2 n$. Il existe $R(x) \in \mathcal{O}_{K}[x]$ tel que $\widetilde{R}(x)^{2}-c_{2 n}(x) \in s_{0}(x) k[x]$. Posons $y_{1}=y+R(x) S_{0}(x)^{n}$. Alors $\left\{1, y_{1}\right\}$ est encore une base, et on a $\delta\left(p_{0}, y_{1}\right)>\delta\left(p_{0}, y\right)$. On continue le raisonnement ci-dessus avec $y_{1}$. Au bout d'un nombre fini de pas, on trouve une base qui donne $\delta\left(p_{0}\right)$. 
On voit de cette manière que si $p_{0}$ est rationnel (ou même quadratique) sur $k$, alors $\delta\left(p_{0}\right)$ est atteinte pour une base standard $\{1, y\}$. Cela est faux en général.

\subsection{Multiplicités dans $W$.}

Soit $r \geq 1$ un entier. Soit $S_{0}(x) \in \mathcal{O}_{K}[x]$ unitaire dont l'image dans $k[x]$ engendre $\mathfrak{m}_{q_{0}}$. Considérons l'anneau $\mathcal{O}_{K}[x, v]$ avec $S_{0}(x)^{r} v=t$. Pour tout $H(x) \in \mathcal{O}_{K}[x]$, on note $\lambda\left(p_{0}, H\right)$ le plus grand entier $n$ tel que $H(x) \in S_{0}(x)^{n} \mathcal{O}_{K}[x, v]$. Par convention, $\lambda_{r}\left(p_{0}, 0\right)=+\infty$. Le nombre $\lambda_{r}\left(p_{0}, H\right)$ est aussi le plus plus grand entier $n$ tel que $H(x) \in\left(t, S_{0}(x)^{r}\right)^{n} \mathcal{O}_{K}[x]$, il est donc indépendant du choix de $S_{0}(x)$. On peut le déterminer de la façon suivante : soit

$$
H(x)=\sum_{i \geq 0} c_{i}(x) S_{0}(x)^{i}, \quad c_{i}(x) \in \mathcal{O}_{K}[x], \operatorname{deg} c_{i}(x)<\operatorname{deg} S_{0}(x)
$$

l'écriture de $H(x)$ dans la base $S_{0}(x)$-adique. Alors

$$
\lambda_{r}\left(p_{0}, H\right)=\min \left\{i+r \nu\left(c_{i}\right) \mid i \geq 0\right\} .
$$

Pour toute base $\{1, y\}$ de $B$ sur $\mathcal{O}_{K}[x]$, on note

$$
\lambda_{r}\left(p_{0}, y\right)=\min \left\{2 \lambda_{r}\left(p_{0}, Q\right), \lambda_{r}\left(p_{0}, P\right)\right\} .
$$

Définition 10. Soit $r \geq 1$, soit $p_{0} \in W_{k}$. La multiplicité $\lambda_{r}\left(p_{0}\right)$ du point $p_{0}$ dans $W$ est le maximum des $\lambda_{r}\left(p_{0}, y\right)$ pour toutes les bases $\{1, y\}$ de $B$ sur $\mathcal{O}_{K}[x]$. On verra (§6.3) que $\lambda_{r}\left(p_{0}\right)$ ne dépend pas du choix du générateur $x$.

On note pour simplifier $\lambda\left(p_{0}\right)=\lambda_{1}\left(p_{0}\right)$. La multiplicité au sens classique de l'anneau local $\mathcal{O}_{W, p_{0}}$ est $\mu\left(W, p_{0}\right)=1$ si $W$ est régulier en $p_{0}$, et $\mu\left(W, p_{0}\right)=2$ sinon. Soit $R \subset W$ le diviseur de ramification de $W \rightarrow Z:=W /\langle\sigma\rangle$ (donc $R=$ $\left.V\left(\operatorname{Ann} \Omega_{W / Z}^{1}\right)\right)$. Alors si $\operatorname{car}(k) \neq 2$ et si $p_{0} \in R$, on peut interpréter $\lambda\left(p_{0}\right)$ comme étant la multiplicité $\mu\left(R, p_{0}\right)$ de l'anneau local de $R$ en $p_{0}$. Par contre, si car $(k)=2$, $\lambda\left(p_{0}\right)$ ne dépend pas que de $R$.

Lemme 7. Soient $W$ un modèle de Weierstrass de $C, p_{0} \in W_{k}$ un point fermé. On a les propriétés suivantes :

(a) Le point $p_{0}$ est lisse sur $k$ si et seulement si $\lambda\left(p_{0}\right)=0$. Il est régulier dans $W$ si et seulement si $\lambda\left(p_{0}\right) \leq 1$.

(b) Soient $v=t / S_{0}(x)^{r}, B_{r}$ la clôture intégrale de $\mathcal{O}_{K}[x, v]$ dans $K(C)$, et $l=$ $\left[\lambda_{r}\left(p_{0}\right) / 2\right]$. Si $y \in B$ est tel que $\lambda_{r}\left(p_{0}, y\right)=\lambda_{r}\left(p_{0}\right)$, alors $\left\{1, y / S_{0}(x)^{l}\right\}$ est une base de $B_{r}$ sur $\mathcal{O}_{K}[x, v]$ (en tant que module).

(c) Il existe une base $\{1, y\}$ de $B$ telle que $\lambda_{s}\left(p_{0}, y\right)=\lambda_{s}\left(p_{0}\right)$ pour tout $s \leq r$.

(d) Soit $B^{\prime}$ la clôture intégrale de $\mathcal{O}_{K}\left[x, S_{0}(x) / t\right]$ dans $K(C)$, soit y tel que $\lambda\left(p_{0}\right)=\lambda\left(p_{0}, y\right)$. Alors $\left\{1, y / t^{\left[\lambda\left(p_{0}\right) / 2\right]}\right\}$ est une base de $B^{\prime}$ sur $\mathcal{O}_{K}\left[x, S_{0}(x) / t\right]$.

(e) Posons $\epsilon=0$ si $W_{k}$ est réduit et $\epsilon=1$ sinon. Alors pour tout $1 \leq s \leq r$, on $a$

$$
\min \left\{1, \delta\left(p_{0}\right)\right\} \leq \lambda_{s}\left(p_{0}\right)-s \epsilon \leq \lambda_{r}\left(p_{0}\right)-r \epsilon \leq \delta\left(p_{0}\right), \quad s \lambda_{r}\left(p_{0}\right) \leq r \lambda_{s}\left(p_{0}\right) .
$$

De plus, si $r \geq \delta\left(p_{0}\right)$, alors $\lambda_{r}\left(p_{0}\right)-r \epsilon=\delta\left(p_{0}\right)$.

(f) L'une des deux inégalités suivantes est toujours vérifiée suivant la nature de $W_{k}$ :

$$
\sum_{p \in W_{k}}[k(p): k](\lambda(p)-\epsilon) \leq 2 g+2
$$




$$
\sum_{p \in W_{k}}[k(p): k](\lambda(p)-1) \leq 2 g .
$$

Preuve. (a) résulte immédiatement du lemme 5.

(b) Soit $z=y / S_{0}(x)^{l}$. Posons $a=Q(x) / S_{0}(x)^{l}, b=-P(x) / s_{0}(x)^{2 l}$. Alors $a, b \in \mathcal{O}_{K}[x, v]$ et $z^{2}+a z+b=0$, donc $z \in B_{r}$. Considérons la sous-algèbre $D:=\mathcal{O}_{K}[x, v, z]$ de $B_{r}$. On veut montrer que $D=B_{r}$. Comme

$$
D \otimes K=K\left[x, 1 / S_{0}(x), y\right]=B_{r} \otimes K
$$

il suffit de montrer que $D$ est normal. L'algèbre $D$ est d'intersection complète sur $\mathcal{O}_{K}$, déterminée par les deux relations $S_{0}(x)^{r} v=t, z^{2}+a z+b=0$, et on a $\operatorname{dim} D=2$. D'après un théorème de Serre ([Mat], Theorem 39, page 125), il suffit de montrer que Spec $D$ est normal en codimension 1, ou encore qu'il est normal aux points génériques de la fibre spéciale Spec $D \otimes k$. Soient $\xi$ un tel point et $\mathfrak{p}$ l'idéal premier de $D$ correspondant à $\xi$.

On a $t \in \mathfrak{p}$. Si $v \in \mathfrak{p}$, alors $D_{\mathfrak{p}}=B_{\mathfrak{p} \cap B}$ est normal. Supposons donc $v \notin \mathfrak{p}$. Alors $S_{0}(x) \in \mathfrak{p}$. Il est facile de vérifier que si $\lambda_{r}\left(p_{0}\right)$ est impair, alors $\mathfrak{p} D_{\mathfrak{p}}$ est engendré par $z$ et que $S_{0}(x) D_{\mathfrak{p}}=z^{2} D_{\mathfrak{p}}$. Si $\lambda\left(p_{0}\right)$ est pair, on a $D /\left(S_{0}(x)\right)=k\left(q_{0}\right)[v, z]$. On montre comme dans le lemme 2 que c'est une algèbre réduite. Il suit que $\mathfrak{p} D_{\mathfrak{p}}$ est engendré par $S_{0}(x)$. Dans tous les cas, $\mathfrak{p} D_{\mathfrak{p}}$ est principal. Donc $D$ est normal. On voit de plus que la parité de $\lambda_{r}\left(p_{0}\right)$ est déterminée par la structure de l'algèbre $D$.

(c) On peut supposer $\operatorname{car}(k)=2$. On montre par récurrence sur $r$ la propriété suivante : il existe une base $\{1, y\}$ de $B$ telle que pour tout $s \leq r$, on ait ou bien $2 \lambda_{s}\left(p_{0}, Q\right) \leq \lambda_{s}\left(p_{0}, P\right)$, ou bien (lorsque l'on écrit $P(x)=\sum_{i} c_{i}(x) S_{0}(x)^{i}$ dans la base $S_{0}(x)$-adique) $2 i+s \nu\left(c_{2 i}\right)>\lambda_{s}\left(p_{0}, P\right)$ pour tout $i$ tel que $\nu\left(c_{2 i}\right) \in \mathbb{N}$ (voir la méthode de calcul de $\lambda_{r}\left(p_{0}\right)$ plus loin).

Soit donc $\{1, y\}$ une base de $B$ construite comme ci-dessus si $\operatorname{car}(k)=2$ et telle que $Q(x)=0$ si $\operatorname{car}(k) \neq 2$. On montre exactement comme dans (b) que pour tout $s \leq r, \mathcal{O}_{K}\left[x, v, y / S_{0}(x)^{l_{s}}\right]=B_{s}$ où $l_{s}=\left[\lambda_{s}\left(p_{0}, y\right) / 2\right]$. Cela implique que $\left[\lambda_{s}\left(p_{0}\right) / 2\right]=\left[\lambda_{s}\left(p_{0}, y\right) / 2\right]$. D'autre part, les parités des $\lambda_{s}\left(p_{0}\right)$ et $\lambda_{s}\left(p_{0}, y\right)$ étant déterminées de la même façon par la structure de $B_{s}$, on a l'égalité $\lambda_{s}\left(p_{0}\right)=$ $\lambda_{s}\left(p_{0}, y\right)$.

(d) Cela se démontre de la même manière que (b).

(e) Il suffit de vérifier les inégalités sur les polynômes dans $\mathcal{O}_{K}[x]$, ce qui est immédiat.

(f) Cela résulte du lemme 6 (c)-(e) et de l'inégalité $\lambda(p)-\epsilon \leq \delta(p)$.

Détermination effective de $\lambda_{r}\left(p_{0}\right)$.

Supposons $p_{0} \in \operatorname{Spec} B$. Soit $\{1, y\}$ une base de $B$. Si $\operatorname{car}(k) \neq 2$, alors $\lambda_{r}\left(p_{0}\right)=$ $\lambda_{r}\left(p_{0}, 4 P+Q^{2}\right)$.

Supposons $\operatorname{car}(k)=2$. Si $2 \lambda_{r}\left(p_{0}, Q\right) \leq \lambda_{r}\left(p_{0}, P\right)$ ou si $\lambda_{r}\left(p_{0}, P\right)$ est impair, alors $\lambda_{r}\left(p_{0}\right)=\lambda_{r}\left(p_{0}, y\right)$. Sinon, écrivons $P(x)$ dans la base $S_{0}(x)$-adique

$$
P(x)=\sum_{j} c_{j}(x) S_{0}(x)^{j} .
$$

S'il existe $i$ tel que $i+r \nu\left(c_{i}\right)=\lambda_{r}\left(p_{0}, P\right)$ et que $\nu\left(c_{i}\right)$ soit impair, on a encore $\lambda_{r}\left(p_{0}\right)=\lambda_{r}\left(p_{0}, y\right)$. Sinon, pour tout indice $2 m$ tel que $2 m+r \nu\left(c_{2 m}\right)=\lambda_{r}\left(p_{0}, P\right)$ et que $\nu\left(c_{2 m}\right)$ soit pair, on prend $b_{m}(x) \in \mathcal{O}_{K}[x]$ tel que $\widetilde{b}_{m}(x)-\widetilde{c}_{2 m}^{\prime}(x) \in \widetilde{S}_{0}(x) k[x]$, où $c_{2 m}^{\prime}(x)=c_{2 m}(x) / t^{\nu\left(c_{2 m}\right)}$. Posons $y_{1}=y+\sum_{m} t^{\nu\left(c_{2 m}\right) / 2} b_{m}(x) S_{0}(x)^{m}$. On a $\lambda_{r}\left(p_{0}, y_{1}\right)>\lambda_{r}\left(p_{0}, y\right)$. On poursuit la même opération avec $y_{1}$. 
Remarque 9 . On voit de cette manière que si $\left[k\left(p_{0}\right): k\right] \leq 2$, alors il existe une base standard satisfaisant l'énoncé du lemme 7 (c). Cela est faux en général.

6.3 Interprétation des multiplicités via les faisceaux dualisants.

On va interpréter les multiplicités $\lambda_{r}\left(p_{0}\right)$ de façon géométrique. On verra en particulier que ce nombre ne dépend pas du choix d'un générateur $x$.

Fixons $r \geq 1$. Notons encore par $\mathfrak{m}_{q_{0}}$ le faisceau d'idéaux sur $W /\langle\sigma\rangle$ correspondant au point $q_{0}$. Soit $Z_{r} \rightarrow W /\langle\sigma\rangle$ l'éclatement de $W /\langle\sigma\rangle$ de centre $t \mathcal{O}_{W /\langle\sigma\rangle}+\mathfrak{m}_{q_{0}}^{r}$, soit $Y_{r} \rightarrow Z_{r}$ la normalisation de $Z_{r}$ dans $C$. Alors il existe un unique morphisme $\phi_{r}: Y_{r} \rightarrow W$ rendant le diagramme suivant commutatif

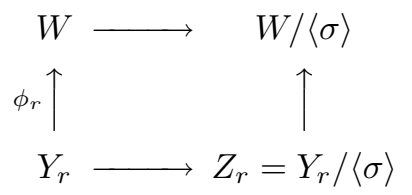

La fibre spéciale de $Z_{r}$ est la réunion d'une composante irréductible de multiplicité 1 et d'une composante de multiplicité $r$. Ces dernières se coupent au point de $\operatorname{Spec} \mathcal{O}_{K}[x, v]$ (avec les notations du lemme $7(\mathrm{~b})$ ) correspondant à l'idéal maximal $\left(t, S_{0}(x), v\right)$.

Notons que si $\lambda\left(p_{0}\right) \geq 2, Y_{1} \rightarrow W$ est aussi la normalisation de l'éclatement de $W$ de centre $p_{0}$.

Rappelons la définition suivante :

Définition 11. Soit $\phi: S \rightarrow T$ un morphisme propre birationnel de schémas connexes normaux. Soit $F$ un fermé pur de codimension 1 dans $T$. Alors le transformé strict de $F$ dans $S$ est l'adhérence $\overline{\left\{\phi^{-1}(\Xi)\right\}}$ dans $S$, où $\Xi$ est la réunion des points génériques de $F$. Notons que $\phi$ induit un isomorphisme d'un voisinage de $\phi^{-1}(\Xi)$ sur un voisinage de $\Xi$.

Lemme 8. Soient $r \geq 1, p_{0} \in W_{k}$ et $\phi_{r}: Y_{r} \rightarrow W$ comme ci-dessus.

(a) Soit $\Gamma$ une composante irréductible de $\phi_{r}^{-1}\left(p_{0}\right)$. Soit $\mathrm{m}(\Gamma)$ sa multiplicité dans $Y_{r}$. Alors $\mathrm{m}(\Gamma)=r$ si $\lambda_{r}\left(p_{0}\right)$ est pair et $\mathrm{m}(\Gamma)=2 r$ sinon.

(b) Soient $\omega_{Y_{r}}, \omega_{W}$ les faisceaux dualisants sur $\mathcal{O}_{K}$ respectivement de $Y_{r}$ et de $W$. Soit $\xi$ le point générique de $\Gamma$. Alors on a un isomorphisme canonique

$$
\left(\left(\phi_{r}^{*} \omega_{W}\right)^{\vee} \otimes \omega_{Y_{r}}\right)_{\xi}^{\otimes r} \simeq t^{l-r} \mathcal{O}_{Y_{r}, \xi}
$$

En particulier, $\lambda_{r}\left(p_{0}\right)$ ne dépend pas du choix de $x$.

(c) Soit $p_{0, r} \in Y_{r}$ un point au-dessus de $p_{0}$ et appartenant au transformé strict de $W_{k}$ dans $Y_{r}$. Alors $p_{0, r}$ est régulier dans $Y_{r}$ si et seulement si $\left[\lambda_{r}\left(p_{0}\right) / 2\right]=$ $\left[\lambda_{r+1}\left(p_{0}\right) / 2\right]$.

(d) Si $W_{k}$ n'est pas réduit, alors l'égalité ci-dessus implique que $\delta\left(p_{0}\right)=\lambda_{r}\left(p_{0}\right)-r$.

Preuve. (a) Soit $\xi$ le point générique de $\Gamma$, soit $\nu_{\xi}$ la valuation de $K(C)$ associé à l'anneaux de valuation discrète $\mathcal{O}_{Y_{r}, \xi}$, alors par définition $\mathrm{m}(\Gamma)=\nu_{\xi}(t)=$ $r \nu_{\xi}\left(S_{0}(x)\right)$. De plus, on a vu dans la preuve du lemme 7 (c) que $\nu\left(S_{0}(x)\right)=1$ ou 2 suivant que $\lambda_{r}\left(p_{0}\right)$ est pair ou non. D'où le résultat.

(b) Conservons les notations du lemme 7 (b). Soit $y \in B$ tel que $\lambda_{r}\left(p_{0}, y\right)=$ $\lambda_{r}\left(p_{0}\right)$, soit $z=y / S_{0}(x)^{l}$. Notons $U=\operatorname{Spec} B$ et $U_{r}=\operatorname{Spec} B_{r}$. On a $\xi \in U_{r}$, $\phi_{r}^{-1}(U)=U_{r}$ et (voir par exemple [Liu2], lemme 2)

$$
\left.\omega_{W}(U)=B d x /(2 y+Q(x)), \quad \omega_{Y_{r}}\left(U_{r}\right)=B_{r} S_{0}(x)^{-r} d x /\left(2 z+Q(x) / S_{0}(x)^{l}\right)\right) .
$$


D'où

$$
\left(\left(\phi_{r}^{*} \omega_{W}\right)^{\vee} \otimes \omega_{Y_{r}}\right)\left(U_{r}\right)=S_{0}(x)^{l-r} B_{r} .
$$

Comme $v$ n'est pas identiquement nulle sur $\Gamma$, on a $S_{0}(x)^{r} \mathcal{O}_{Y_{r}, \xi}=t \mathcal{O}_{Y_{r}, \xi}$. D'où le résultat.

(c) Supposons $\operatorname{car}(k)=2$, le cas contraire étant plus simple à traiter. On garde les notations du lemme 7 . Le point $p_{0, r}$ correspond à un idéal maximal $\mathfrak{m}_{p_{0, r}}$ de $B_{r}$ contenant $S_{0}(x)$ et $v$. Si $Q(x) / S_{0}(x)^{l} \notin\left(S_{0}(x), v\right)$, alors $Y_{r} \rightarrow Z_{r}$ est non-ramifié en $p_{0, r}$, donc $p_{0, r}$ est régulier dans $Y_{r}$. De plus, on a dans ce cas-là

$$
\lambda_{r+1}\left(p_{0}, Q\right)=\lambda_{r}\left(p_{0}, Q\right) \leq \lambda_{r}\left(p_{0}\right) / 2 \leq \lambda_{r+1}\left(p_{0}, P\right) / 2
$$

donc $\lambda_{r+1}\left(p_{0}\right)=\lambda_{r+1}\left(p_{0}, y\right)=\lambda_{r}\left(p_{0}\right)$. On suppose dans la suite que $Q(x) / S_{0}(x)^{l} \in$ $\left(S_{0}(x), v\right)$. Cela implique que $\lambda_{r+1}\left(p_{0}, Q\right) \geq l+1$. Quitte à faire une translation sur $y$, on peut supposer que $P(x) / S_{0}(x)^{2 l} \in\left(S_{0}(x), v\right)$. Donc $\lambda_{r+1}\left(p_{0}, P\right) \geq 2 l+1$ et $y / S_{0}(x)^{l} \in \mathfrak{m}_{p_{0, r}}$. Par conséquent, $Y_{r}$ est régulier en $p_{0, r}$ si et seulement si $P(x) / S_{0}(x)^{2 l} \notin\left(S_{0}(x), v\right)^{2}$, ce qui équivaut à $\lambda_{r+1}\left(p_{0}, P\right)=2 l+1$. L'équivalence : $Y_{r}$ régulier en $p_{0, r} \Longleftrightarrow\left[\lambda_{r+1}\left(p_{0}\right) / 2\right]=\left[\lambda_{r}\left(p_{0}\right) / 2\right]$ est alors immédiate.

(d) Avec les notations ci-dessus, il existe (en écrivant $P(x)=\sum_{i} a_{i}(x) S_{0}(x)^{i}$ ) un $i_{0}$ tel que $\nu\left(a_{i_{0}}\right)=1$ et $i_{0}+r \nu\left(a_{i_{0}}\right)=2 l$. On a donc $\lambda_{r}\left(p_{0}\right)=2 l$, et $\delta\left(p_{0}\right)=$ $i_{0}=2 l-r$.

Remarque 10. En utilisant le lemme ci-dessus, on peut montrer que le faisceau quotient $\omega_{W} / \phi_{1 *} \omega_{Y_{1}}$, qui est à support en $p_{0}$, est isomorphe au faisceau associé à $B / I$, où $I$ est l'idéal $\left\{R(x)+T(x) y \in B \mid \lambda\left(p_{0}, R\right) \geq\left[\lambda\left(p_{0}\right) / 2\right]-1\right\}$. Supposons que $p_{0} \in W_{k}$ soit une singularité rationnelle de $W$ (voir [Art], p. 268). Donc $\phi_{1 *}\left(\omega_{Y_{1}}\right)=\omega_{W}$. Il suit alors que $\lambda\left(p_{0}\right) \leq 3$. Cela découle aussi du fait que l'éclatement d'une singularité rationnelle donne un schéma normal ([Art], Theorem $4.9)$.

\section{Critère de minimalité}

Soit $W$ un modèle de Weierstrass de $C$ sur $\mathcal{O}_{K}$. On va définir pour tout point rationnel $p_{0} \in W_{k}(k)$ une nouvelle multiplicité $\lambda^{\prime}\left(p_{0}\right)$. Cela nous permettra de donner un critère de minimalité de $W$ (corollaire 2 ) en fonction du maximum des multiplicités $\lambda^{\prime}(p)$ et $\lambda(p)$ pour $p \in W_{k}(k)$. On étudie aussi le comportement du discriminant minimal $\nu(C)$ vis-à-vis d'un changement de base étale (proposition 4 ). Enfin, on montre une borne absolue de $\nu(C)$ sous certaines hypothèses ( $\S 7.3)$.

\subsection{Critère de minimalité.}

Définition 12. Soient $W$ un modèle de Weierstrass de $C$ et $p_{0} \in W_{k}(k)$. On note $W\left(p_{0}\right)$ l'unique modèle de Weierstrass de $C$ tel que $\mathrm{d}\left(W, W\left(p_{0}\right)\right)=1$ et que $p_{0} \in W\left(p_{0}\right) \wedge W$ (voir déf. 7 ).

Avec les notations du $\S 5$, on a $\operatorname{deg} S_{0}(x)=1$ et $W\left(p_{0}\right)$ est le modèle de Weierstrass de $C$ associé à $S_{0}(x) / t$. D'autre part, on a un morphisme canonique $Y_{1} \rightarrow$ $W\left(p_{0}\right)$ (voir $\S 6.3$ pour la définition de $Y_{1}$ ) qui induit un isomorphisme d'un voisinage des points génériques de $\phi_{1}^{-1}\left(p_{0}\right)$ sur son image.

Définition 13. Soient $W, p_{0}$ comme ci-dessus. On note $\lambda^{\prime}\left(p_{0}\right)$ l'entier

$$
\lambda^{\prime}\left(p_{0}\right):=\max \left\{\lambda(p) \mid p \in W\left(p_{0}\right)_{k}(k), p \notin W\left(p_{0}\right) \wedge W\right\} .
$$


Par convention le maximum d'un ensemble vide est $-\infty$. Plus généralement, pour tout entier $d \geq 1$, on peut définir $\lambda^{d}\left(p_{0}\right)$ comme étant le maximum

$$
\max \left\{\lambda(p) \mid d\left(W^{\prime}, W\right) \leq d, p_{0} \in W^{\prime} \wedge W, p \in W_{k}^{\prime}(k), p \notin W^{\prime} \wedge W\right\} .
$$

Soit $B^{\prime}$ la clôture intégrale de $\mathcal{O}_{K}\left[x, S_{0}(x) / t\right]$ dans $K(C)$. Alors $\lambda^{\prime}\left(p_{0}\right)$ est le maximum des multiplicités $\lambda(p)$ (calculées dans $\left.W\left(p_{0}\right)\right)$ pour $p \in \operatorname{Spec}\left(B^{\prime} \otimes k\right)$ rationnels sur $k$.

Lemme 9. Soient $W$ un modèle de Weierstrass de $C$ sur $\mathcal{O}_{K}, p_{0} \in W_{k}(k)$ et $W\left(p_{0}\right)$ le modèle de Weierstrass associé à $p_{0}$ comme ci-dessus.

(a) On a

$$
\nu\left(\Delta_{W\left(p_{0}\right)}\right)-\nu\left(\Delta_{W}\right)=2(2 g+1)\left(g+1-2\left[\lambda\left(p_{0}\right) / 2\right]\right) .
$$

(b) Posons $\epsilon=0$ si $W_{k}$ est réduit et $\epsilon=1$ sinon. Soit $p_{0}^{\prime} \in W\left(p_{0}\right) \cap\left(W\left(p_{0}\right) \wedge W\right)$, alors

$$
\lambda\left(p_{0}^{\prime}\right)=2 g+2-2\left[\lambda\left(p_{0}\right) / 2\right]+\epsilon, \quad \lambda_{2}\left(p_{0}^{\prime}\right) \geq \lambda_{2}\left(p_{0}\right)-2\left[\lambda\left(p_{0}\right) / 2\right]+2 g+2 .
$$

(c) On a $\lambda^{\prime}\left(p_{0}\right) \leq 2\left[\left(\lambda\left(p_{0}\right)+1\right) / 2\right]-\epsilon$.

(d) L'une des deux inégalités

$$
\sum_{p \in W\left(p_{0}\right)_{k} \backslash\left(W\left(p_{0}\right) \wedge W\right)} \delta(p)[k(p): k] \leq \lambda\left(p_{0}\right)-\epsilon
$$

ou

$$
\sum_{p \in W\left(p_{0}\right)_{k} \backslash\left(W\left(p_{0}\right) \wedge W\right)}(\delta(p)-1)[k(p): k] \leq \lambda\left(p_{0}\right)-1-\epsilon
$$

est toujours vérifiée. De plus, la première inégalité est toujours une égalité si $\lambda\left(p_{0}\right)$ est impair ou si $\operatorname{car}(k) \neq 2$.

Preuve. On garde les notations du $\S 5$. On peut supposer $S_{0}(x)=x$. Notons $u=x / t$ et $l=\left[\lambda\left(p_{0}\right) / 2\right]$. Soit $\{1, y\}$ une base standard de $B$ telle que $\lambda\left(p_{0}, y\right)=\lambda\left(p_{0}\right)$ (voir remarque 9$)$.

(a) Soit $z=y / t^{l}$. Le modèle $W^{\prime}$ admet d'après le lemme 7 (d) un ouvert affine d'équation

$$
z^{2}+\left(Q(t u) / t^{l}\right) z=P(t u) / t^{2 l} .
$$

On en déduit immédiatement la différence $\nu\left(\Delta_{W\left(p_{0}\right)}\right)-\nu\left(\Delta_{W}\right)$ grâce à la formule à la fin du $\S 2$.

(b) Le point $p_{0}^{\prime}$ est rationnel sur $k$. Le modèle $W\left(p_{0}\right)\left(p_{0}^{\prime}\right)$ associé à $p_{0}^{\prime} \in W\left(p_{0}\right)$ n'est autre que $W$. Donc

$$
\nu\left(\Delta_{W}\right)-\nu\left(\Delta_{W\left(p_{0}\right)}\right)=2(2 g+1)\left(g+1-2\left[\lambda\left(p_{0}^{\prime}\right) / 2\right]\right) .
$$

Il suit que $2\left[\lambda\left(p_{0}^{\prime}\right) / 2\right]=2 g+2-2 l$. Comme $\lambda\left(p_{0}^{\prime}\right)=2\left[\lambda\left(p_{0}^{\prime}\right) / 2\right]+\epsilon$, on a l'égalité annoncée. Soit $\{1, y\}$ une base standard de $B$ telle que $\lambda_{i}\left(p_{0}, y\right)=\lambda_{i}\left(p_{0}\right)$ pour $i=1,2$ (voir remarque 9 ). Soient $v=1 / u, w=y /\left(t^{l} u^{g+1}\right)$. Alors le point $p_{0}^{\prime}$ est un zéro de $v$. Donc $\lambda_{2}\left(p_{0}^{\prime}\right) \geq \lambda_{2}\left(p_{0}^{\prime}, w\right)=\lambda_{2}\left(p_{0}\right)-2 l+2 g+2$.

(c) Cela résulte du lemme 7 (f) compte tenu de l'égalité sur $\lambda\left(p_{0}^{\prime}\right)$ ci-dessus.

(d) Soit $\{1, y\}$ une base standard de $B$. Quitte à faire une translation sur $y$, on peut supposer que l'on a aussi $\delta\left(p_{0}, y\right)=\delta\left(p_{0}\right)$. Posons $R(u)=Q(t u) / t^{l}, T(u)=$ $P(t u) / t^{2 l}$ et $T_{1}(u)=T(u) / t$ si $\nu(R) \geq 1, \nu(T) \geq 1$. On a alors $\operatorname{deg} \widetilde{R}(u) \leq l-\epsilon$, $\operatorname{deg} \widetilde{T}(u) \leq 2 l-\epsilon$ et $\operatorname{deg} \widetilde{T}_{1}(u) \leq 2 l+1-\epsilon$ s'il y a lieu de considérer $T_{1}(u)$. Les assertions de (d) se montrent comme pour le lemme 6 (c)-(e). 
Proposition 3. Soient $W$ un modèle de Weierstrass de $C$ sur $\mathcal{O}_{K}, p_{0} \in W_{k}$. Considérons les propriétés suivantes :

(i) Pour tout modèle de Weierstrass $W^{\prime}$ non isomorphe à $W$ et tel que $p_{0} \in$ $W^{\prime} \wedge W$ (voir déf. 7 ), on a $\nu\left(\Delta_{W^{\prime}}\right) \geq \nu\left(\Delta_{W}\right)$

(ii) On a les inégalités $\lambda\left(p_{0}\right) \leq 2[(g+1) / 2]+1$ et $\lambda^{\prime}\left(p_{0}\right) \leq g+2$;

(iii) Pour tout modèle de Weierstrass $W^{\prime}$ comme dans (i), on a $\nu\left(\Delta_{W^{\prime}}\right)>\nu\left(\Delta_{W}\right)$;

(iv) On a les inégalités $\lambda\left(p_{0}\right) \leq 2[g / 2]+1$ et $\lambda^{\prime}\left(p_{0}\right) \leq g+1$.

Alors (i) $\Longleftrightarrow$ (ii); et (iii) $\Longleftrightarrow$ (iv).

Preuve. Montrons seulement (i) $\Longleftrightarrow$ (ii). L'autre équivalence se démontre de la même façon.

Supposons d'abord que les inégalités de (ii) ne sont pas simultanément satisfaites. D'après le lemme 9 (c), on a $\lambda\left(p_{0}\right) \geq g+2$. Si $g$ est pair ou si $\lambda\left(p_{0}\right) \geq g+3$, on a $\nu\left(\Delta_{W\left(p_{0}\right)}\right)<\nu\left(\Delta_{W}\right)$ d'après le lemme 9 (a). Supposons $g$ impair et $\lambda\left(p_{0}\right)=g+2$. Alors par hypothèse $\lambda^{\prime}\left(p_{0}\right) \geq g+3$. Soit $p_{1} \in W\left(p_{0}\right)(k) \backslash\left(W\left(p_{0}\right) \wedge W\right)$ tel que $\lambda\left(p_{1}\right)=\lambda^{\prime}\left(p_{0}\right)$, soit $W^{\prime}$ le modèle $W\left(p_{0}\right)\left(p_{1}\right)$ associé à $p_{1}$. Il suit du lemme 9 (a) que $\nu\left(\Delta_{W^{\prime}}\right)<\nu\left(\Delta_{W\left(p_{0}\right)}\right)=\nu\left(\Delta_{W}\right)$. Ce qui montre que (i) implique (ii).

Montrons maintenant la réciproque. Supposons les inégalités de (ii) satisfaites. Soit $d=d\left(W, W^{\prime}\right)$. Il existe $x, u \in K(C)^{\langle\sigma\rangle}$ tels que $W /\langle\sigma\rangle=\mathbb{P}_{x}^{1}, W^{\prime} /\langle\sigma\rangle=\mathbb{P}_{u}^{1}$, $x=t^{d} u$, et que $x\left(p_{0}\right)=0$. Pour tout $0 \leq i \leq d$, soit $W^{i}$ le modèle de Weierstrass associé à $x / t^{i}$. Notons $p_{i}$ un point de $W^{i}$ correspondant à $\left(x / t^{i}\right)=t=0$. Alors $W^{0}=W, W^{d}=W^{\prime}$ et $W^{i+1}=W^{i}\left(p_{i}\right)$.

Supposons que $g$ est impair. On montre par récurrence sur $i$ et en utilisant le lemme 8 (a) et le lemme 9 (c) que $p_{i}$ satisfait les inégalités de (ii) pour tout $i \leq d$. Ce qui implique que $\nu\left(\Delta_{W^{\prime}}\right)=\nu\left(\Delta_{W^{d}}\right) \geq \nu\left(\Delta_{W^{d-1}}\right) \geq \ldots \geq \nu\left(\Delta_{W^{0}}\right)=\nu\left(\Delta_{W}\right)$.

Supposons maintenant $g$ pair. On a

$$
\nu\left(\Delta_{W^{2}}\right)-\nu\left(\Delta_{W}\right)=4(2 g+1)\left(g+1-\left[\lambda\left(p_{0}\right) / 2\right]-\left[\lambda\left(p_{1}\right) / 2\right]\right) .
$$

Comme $\left[\lambda\left(p_{0}\right) / 2\right]+\left[\lambda\left(p_{1}\right) / 2\right] \leq[(g+1) / 2]+[(g+2) / 2]=g+1$, on a $\nu\left(\Delta_{W^{2}}\right) \geq$ $\nu\left(\Delta_{W}\right)$. D'autre part, on montre comme dans le cas $g$ impair que les points $p_{2 i}$ satisfont les inégalités de (ii). Donc $\nu\left(\Delta_{W^{2 i+1}}\right), \nu\left(\Delta_{W^{2 i+2}}\right) \geq \nu\left(\Delta_{W^{2 i}}\right)$. D'où $\nu\left(\Delta_{W^{\prime}}\right) \geq \nu\left(\Delta_{W}\right)$.

Corollaire 2. Soit $W$ un modèle de Weierstrass de $C$ sur $\mathcal{O}_{K}$. Alors on a les propriétés suivantes :

(a) $W$ est minimal si et seulement si pour tout point $p_{0} \in W_{k}(k)$, on a

$$
\lambda\left(p_{0}\right) \leq 2[(g+1) / 2]+1, \quad \lambda^{\prime}\left(p_{0}\right) \leq g+2 .
$$

(b) W est l'unique modèle de Weierstrass minimal si et seulement si pour tout point $p_{0} \in W_{k}(k)$, on a

$$
\lambda\left(p_{0}\right) \leq 2[g / 2]+1, \quad \lambda^{\prime}\left(p_{0}\right) \leq g+1 .
$$

Corollaire 3. Soit $W$ un modèle de Weierstrass de $C$ sur $\mathcal{O}_{K}$. S'il est régulier, alors c'est l'unique modèle de Weierstrass minimal de $C$ sur $\mathcal{O}_{K}$.

Remarque 11. Il est aisé, en partant d'un modèle de Weierstrass $W$ quelconque, de trouver un modèle de Weierstrass minimal. En effet, on calcule $\lambda\left(p_{0}\right)$ et $\lambda^{\prime}\left(p_{0}\right)$ pour tous les points de $W_{k}(k)$ singuliers dans $W$. S'il existe un $p_{0}$ ne satisfaisant pas les inégalités de prop. 3 (ii), on remplace $W$ par le modèle de Weierstrass $W\left(p_{0}\right)$ 
associé à $p_{0}$, et on recommence avec $W\left(p_{0}\right)$. Lorsque cela n'est plus possible, c'est que le dernier modèle de Weierstrass est minimal.

Notons aussi que si $\lambda\left(p_{0}\right) \leq g+1$, alors $\lambda^{\prime}\left(p_{0}\right) \leq g+2$ en vertu du lemme 9 (c).

Exemple. Soit $C$ la courbe hyperelliptique définie par l'équation $y^{2}=x^{5}+n$, où $n$ est un entier impair tel que $\nu_{p}(n) \leq 3$ pour tout nombre premier $p$. Le discriminant de cette équation est $2^{8} 5^{5} n^{4}$ (voir $\S 2$ ). Soit $W$ le modèle de Weierstrass (sur $\mathbb{Z}$ ) associé à cette équation. Montrons que $W$ est minimal sur $\mathbb{Z}$.

On a $g=2$. Soit $p$ un nombre premier. Si $\nu_{p}(n) \leq 1$, alors $\nu_{p}\left(\Delta_{W}\right)<10=$ $2(2 g+1)$, donc $W$ est minimal sur $\mathbb{Z}_{p}$ (voir $\S 7.3$ ci-dessous). Si $\nu_{p}(n) \geq 2$, alors $W_{p}$ a un unique point singulier de multiplicité $\lambda=\nu_{p}(n) \leq 3$, il est donc minimal d'après le corollaire 2 .

7.2 Changement de base étale.

Soit $\mathcal{O}_{L}$ un anneau de valuation discrète étale (i.e. non-ramifié) sur $\mathcal{O}_{K}$, de corps de fractions $L$. On souhaite comparer le discriminant minimal de $C$ avec celui de $C_{L}:=C \times \operatorname{Spec} K \operatorname{Spec} L$. On note encore par $\nu$ la valuation sur $L$. Elle prolonge celle de $\mathcal{O}_{K}$.

Lemme 10. Soit $W$ un modèle de Weierstrass de $C$ sur $\mathcal{O}_{K}$. Soit $p_{0} \in W_{k}$ un point fermé. Alors pour tout point $r_{0} \in W_{\mathcal{O}_{L}}:=W \times_{\operatorname{Spec} \mathcal{O}_{K}} \operatorname{Spec} \mathcal{O}_{L}$ au-dessus de $p_{0}$, on a $\lambda\left(r_{0}\right)=\lambda\left(p_{0}\right)$.

Preuve. Il est claire d'abord que $W_{\mathcal{O}_{L}}$ est un modèle de Weierstrass de $C_{L}$ sur $\mathcal{O}_{L}$. Comme le revêtement étale d'un schéma normal est normal ([Mil2], I.3, Prop. 3.17 (b), page 27), la construction du morphisme $\phi_{1}: Y_{1} \rightarrow W$ du $\S 6$ commute au changement de base $\operatorname{Spec} \mathcal{O}_{L} \rightarrow \operatorname{Spec} \mathcal{O}_{K}$. Plus précisément, soit $\psi: Y_{1}^{\prime} \rightarrow W_{\mathcal{O}_{L}}$ le morphisme construit comme dans $\S 6.3$ relativement à $r_{0}$. Alors il existe un morphisme $\left(Y_{1}\right)_{\mathcal{O}_{L}} \rightarrow Y_{1}^{\prime}$ qui induit un isomorphisme dans un voisinage de $\psi^{-1}\left(r_{0}\right)$. Comme la formation des faisceaux dualisants commute au changement de base étale, et qu'un tel changement de base ne modifie pas les multiplicités des composantes irréductibles, il résulte du lemme $8(\mathrm{a})$-(b) que $\lambda\left(r_{0}\right)=\lambda\left(p_{0}\right)$.

Proposition 4. Soit $W$ un modèle de Weierstrass minimal de C. Les propriétés suivantes sont équivalentes :

(i) Il existe une extension quadratique non-ramifiée $\mathcal{O}_{L}$ de $\mathcal{O}_{K}$ telle que $W_{\mathcal{O}_{L}}$ ne soit pas minimal.

(ii) Il existe une extension finie non-ramifiée $\mathcal{O}_{L}$ de $\mathcal{O}_{K}$ telle que $W_{\mathcal{O}_{L}}$ ne soit pas minimal.

(iii) L'entier $g$ est pair, et il existe $p_{0} \in W_{k}$ tel que $\left[k\left(p_{0}\right): k\right]=2, \lambda\left(p_{0}\right)=g+2$. De plus, si ces conditions sont vérifiées, $W$ est l'unique modèle minimal de $C$; le discriminant minimal $\nu\left(C_{L}\right)$ sur $\mathcal{O}_{L}$ vérifie

$$
\nu\left(C_{L}\right)=\nu(C)-2(2 g+1)
$$

et ce discriminant minimal $\nu\left(C_{L}\right)$ reste inchangé par toute extension non-ramifiée de $\mathcal{O}_{L}$.

Preuve. Il n'y a rien à démontrer pour (i) $\Longrightarrow$ (ii). Supposons que (ii) est vraie. Les inégalités (ii) de la prop. 3 ne sont pas simultanément satisfaites pour un point rationnel $r_{0}$ de la fibre spéciale de $W_{\mathcal{O}_{L}}$. Soit $p_{0} \in W_{k}$ l'image de $r_{0}$. Alors $\lambda\left(p_{0}\right)=\lambda\left(r_{0}\right) \geq g+2$. De plus, $k\left(p_{0}\right) \neq k$ car sinon $W$ ne serait pas minimal. Il suit du lemme 6 (d)-(e) et du lemme 7 (e) que $\lambda\left(p_{0}\right)=g+2, W_{k}$ n'est pas réduit, 
et que $\left[k\left(p_{0}\right): k\right]=2$. D'après le lemme $9(\mathrm{c})$, on a $\lambda^{\prime}\left(r_{0}\right) \leq g+2$. Donc $g$ doit être pair pour que $W_{\mathcal{O}_{L}}$ ne soit pas minimal. Donc (ii) implique (iii).

Supposons (iii) satisfaite. Soit $\mathcal{O}_{L}=\mathcal{O}_{K}[x] /\left(S_{0}(x)\right)$ (avec les notations du $\S 5$ ). Alors $\mathcal{O}_{L}$ est une extension quadratique non-ramifiée de $\mathcal{O}_{K}$. De plus, si $r_{0} \in W_{\mathcal{O}_{L}}$ est un antécédent de $p_{0}$, alors $r_{0}$ est rationnel sur le corps résiduel de $\mathcal{O}_{L}$. Il suit du corollaire 2 (a) que $W_{\mathcal{O}_{L}}$ n'est pas minimal.

Pour la dernière assertion, après avoir remplacé $\mathcal{O}_{K}$ par $\mathcal{O}_{L}$, on est dans la situation d'un modèle de Weierstrass $W$ tel qu'il existe $p_{0} \in W_{k}(k)$ de multiplicité $\lambda\left(p_{0}\right)=g+2$, et que $W_{k}$ ne soit pas réduit. Soit $W^{\prime}=W\left(p_{0}\right)$ le modèle de Weierstrass associé à $p_{0}$. Alors on a $\nu\left(\Delta_{W^{\prime}}\right)=\nu\left(\Delta_{W}\right)-2(2 g+1)$ d'après lemme 9 (a). En utilisant le lemme 9 (b)-(c) et le fait que $g$ est pair, on voit que $W^{\prime}$ remplit les critères du corollaire 2 (a) et est donc minimal. Comme la fibre spéciale de $W^{\prime}$ est réduite, il est minimal sur toute extension non-ramifiée de $\mathcal{O}_{L}$ d'après ce qui précède.

Remarque 12. La proposition ci-dessus décrit le comportement du discriminant minimal $\nu(C)$ vis-à-vis d'un changement de base étale $\mathcal{O}_{L} / \mathcal{O}_{K}$. La situation devient plus complexe lorsque $\mathcal{O}_{L} / \mathcal{O}_{K}$ est ramifiée. Soient $\nu_{L}$ la valuation normalisée de $L$, $e_{L / K}$ l'indice de ramification. On a facilement $\nu(C) \geq e_{L / K}^{-1} \nu_{L}\left(C_{L}\right)$. Inversement, soit $c=\nu(C)-e_{L / K}^{-1} \nu_{L}\left(C_{L}\right)$. Il semble fort probable que les propriétés suivantes soient vraies :

(a) si $L / K$ est modérément ramifiée, alors $c$ est majorée par une constante dépendant uniquement du genre $g$;

(b) si $L / K$ est sauvagement ramifiée et si $\operatorname{car}(K)=0$, alors $c$ est majorée par une constante dépendant uniquement de $g$ et de l'indice de ramification absolu de $K$;

(c) enfin $c=0$ si le modèle régulier minimal $X$ de $C$ sur $\mathcal{O}_{K}$ (voir $\S 8$ ) est semistable (c'est-à-dire que $X_{k}$ est réduit et que les points singuliers de $X_{k}$ sont des points doubles ordinaires).

La section suivante fournit une réponse à (a) dans un cas partiel. D'autre part, on peut montrer que si $0<\operatorname{car}(K)<2 g+1$, la différence $c$ peut être arbitrairement grande pour $g$ fixé. Il suffit pour cela de prendre pour $C$ des courbes elliptiques ayant potentiellement bonne réduction mais avec de grands conducteurs.

7.3 Le cas de la potentielle bonne réduction.

Soit $W$ un modèle de Weierstrass de $C$. D'après la formule en fin de $\S 2$, si on a $\nu\left(\Delta_{W}\right)<2(2 g+1) \operatorname{pgcd}(g+1,2)$, alors $W$ est minimal. L'inverse est faux, et il n'y a pas de majoration de $\nu(C)$ en terme uniquement de $g$ et de $K$, même si $\operatorname{car}(k)=0$. Cela provient de l'éventualité de la réduction stable singulière. Cependant si $C$ a potentiellement bonne réduction, on a une réciproque partielle.

Remarque 13. Supposons que $C$ a potentiellement bonne réduction et que $\operatorname{car}(k)=$ 0 ou $\operatorname{car}(k)>2 g+1$. Alors le discriminant minimal $\nu(C)$ vérifie

$$
\nu(C) \leq 2(2 g+1)([(g+1) / 2]+1) .
$$

La borne est atteinte par la courbe associée à l'équation $y^{2}=t\left(x^{2 g+2}+t^{2[(g+1) / 2]}\right)$.

Preuve. Soit $W$ un modèle de Weierstrass minimal de $C$. Si $W$ vérifie les conditions de la prop. 4, on peut voir que $C$ n'a pas potentiellement bonne réduction. On peut donc supposer $\mathcal{O}_{K}$ strictement hensélien. Soit $L$ l'extension de $K$ de degré 
minimal sur laquelle $C$ acquiert bonne réduction. L'hypothèse sur car $(k)$ implique que $L$ est modérément ramifiée sur $K$ ([Vie], Satz 2.2). Pour un choix convenable de $x \in K(C)^{\langle\sigma\rangle}$, W est associé à une équation entière $y^{2}=P(x)$ et il existe $\alpha, \beta \in \mathcal{O}_{L}$ tels que le changement de variable $x=\alpha u, y=\beta v$ conduise à une équation $v^{2}=H(u)$ avec $H(u) \in \mathcal{O}_{L}[u]$ et $\operatorname{disc}(H(u)) \in \mathcal{O}_{L}^{*}$.

Soient $n=[L: K], \pi$ une uniformisante de $\mathcal{O}_{L}$. Quitte à changer $u$ et $v$, il existe $r, s \geq 0$, tels que $x=\pi^{r} u, y=\pi^{s} v$. Ecrivons $P(x)=\sum_{i} a_{i} x^{i}$. Alors $2 s=\min \left\{n \nu\left(a_{i}\right)+r i \mid i \geq 0\right\}, P(x)=\pi^{2 s} H(u)$, et $n \nu\left(\Delta_{W}\right)=2(2 g+1)(2 s-(g+1) r)$. Comme l'image de $H(u)$ dans $k[u]$ est un polynôme séparable de degré $\geq 2 g+1$, en utilisant les inégalités sur les multiplicités $\lambda$ (cor. 2), on obtient sans difficulté la majoration souhaitée.

\section{RELATION AVEC LE MODÈLE RÉGULIER MINIMAL}

Soit $X$ le modèle régulier minimal de $C$ sur $\mathcal{O}_{K}$. C'est par définition un schéma régulier, projectif et plat sur $\mathcal{O}_{K}$, à fibre générique $X_{K}$ isomorphe à $C$ et sans diviseur exceptionnel (voir [Chi], §1). Ce modèle est unique à isomorphe près.

Soit $W$ un modèle de Weierstrass de $C$. On note $\widetilde{W} \rightarrow W$ la désingularisation minimale de $W$. Dire que $\widetilde{W}$ est isomorphe à $X$ revient à dire que le transformé strict de $W_{k}$ dans $\widetilde{W}$ (voir $\S 6$, déf. 11) ne contient pas de diviseur exceptionnel.

Lemme 11. Soit $\mathcal{O}_{L}$ un anneau de valuation discrète étale sur $\mathcal{O}_{K}$. Alors $X_{\mathcal{O}_{L}}$ est le modèle régulier minimal de $C_{L}$ sur $\mathcal{O}_{L}$.

Preuve. Le critère de Castelnouvo pour les diviseurs exceptionnels ([Chi], Theorem 3.1) montre que l'on peut remplacer $\mathcal{O}_{K}$ par son complété. On suppose donc $\mathcal{O}_{K}$ complet. Il suffit de montrer que $X \times \widetilde{\mathcal{O}_{L}}$ est minimal, où $\widetilde{\mathcal{O}_{L}}$ et la clôture galoisienne de $\mathcal{O}_{L}$ sur $\mathcal{O}_{K}$. Comme $\widetilde{\mathcal{O}_{L}}$ reste étale sur $\mathcal{O}_{K}$ car celui-ci est complet, on peut supposer que $\mathcal{O}_{L} / \mathcal{O}_{K}$ est galoisien de groupe de Galois $G$.

Notons $S=\operatorname{Spec} \mathcal{O}_{K}$ et $T=\operatorname{Spec} \mathcal{O}_{L}$. Soit $Y$ le modèle régulier minimal de $C_{L}$ sur $T$. Alors $G$ opère sur $Y$ (en tant que $S$-schéma) par l'unicité du modèle régulier minimal. Le schéma $Y$ étant projectif sur $T$ ([Lic2], Theorem 2.8), donc projectif sur $S$, le quotient $Y / G$ existe et est un modèle normal de $C$ sur $S$. Donc $(Y / G) \times{ }_{S} T$ est normal car étale sur $Y / G$ ([Mil2], loc. cit.). Il suit que le morphisme canonique $Y \rightarrow(Y / G) \times{ }_{S} T$ est un isomorphisme (car il est fini et birationnel). Cela implique que $Y \rightarrow Y / G$ est plat, donc $Y / G$ est régulier ([Mat], Theorem 51, page 155). Par conséquent $Y / G$ domine $X$, donc $Y$ domine $X \times{ }_{S} T$, qui est lui-même régulier, on a donc $Y \simeq X \times{ }_{S} T$.

Remarque 14. Les résultats de 7.2 et le lemme ci-dessus restent valables si l'on remplace une extension étale de $\mathcal{O}_{K}$ par le complété de $\mathcal{O}_{K}$ pour la valuation $\nu$.

Proposition 5. Soit $W$ un modèle de Weierstrass minimal de $C$ sur $\mathcal{O}_{K}$. Soient $\widetilde{W} \rightarrow W$ la désingularisation minimale de $W$ et $X$ le modèle régulier minimal de $C$ sur $\mathcal{O}_{K}$. Supposons qu'il existe $p_{0} \in W_{k}$ tel que $\lambda\left(p_{0}\right)=g+2$ sig est pair et $\lambda_{2}\left(p_{0}\right)=2 g+4$ si $g$ est impair. Alors on a les propriétés suivantes :

(a) La fibre spéciale $W_{k}$ n'est pas réduite, et $\widetilde{W}$ n'est pas isomorphe à $X$.

(b) Si g est impair, alors $C$ a exactement deux modèles de Weierstrass minimaux. De plus, les composantes irréductibles de $X_{k}$ sont alors de multiplicités paires. 
Preuve. Supposons d'abord que $g$ est pair. On a $\left[k\left(p_{0}\right): k\right]=2, \lambda\left(p_{0}\right)=g+2$ en vertu du corollaire 2 (a) et du lemme 7 (f). Il résulte alors des lemmes 6 et 7 que $W_{k}$ n'est pas réduit, $\delta\left(p_{0}\right)=g+1, \lambda_{2}\left(p_{0}\right)=g+3$ et que $p_{0}$ est l'unique point singulier de $W$. Soit $\phi_{1}: Y_{1} \rightarrow W$ le morphisme construit au $\S 6.3$ relatif au point $p_{0}$ avec $r=1$. D'après le lemme 8 (c), $Y_{1}$ est régulier dans un voisinage de $\Gamma$, transformé strict de $W_{k}$. Soient $\Gamma_{1}, \cdots, \Gamma_{n}$ les composantes de $\left(Y_{1}\right)_{k}$ qui coupent $\Gamma, \Theta$ l'image de $\Gamma$ dans $Y_{1} /\langle\sigma\rangle$ et $\Theta_{1} \subset Y_{1} /\langle\sigma\rangle$ l'image commune des $\Gamma_{i}$. Alors les $\Gamma_{i}$ et $\Theta_{1}$ sont définis sur $k\left(p_{0}\right)$ et on a $\Gamma_{i} \cdot \Gamma \geq 2, \Theta \cdot \Theta_{1}=2$ (les nombres d'intersection sont calculés sur $k$ ). La formule de projection (voir par exemple [Lan], III, Theorem $4.1)$

$$
\Gamma \cdot \phi_{1}^{*} \Theta_{1}=\left(\phi_{1 *} \Gamma\right) \cdot \Theta_{1}
$$

donne

$$
\sum_{i \geq 1}\left(\Gamma \cdot \Gamma_{i}\right)=\Theta \cdot \Theta_{1}
$$

Cela implique que $n=1$ et que $\Gamma \cdot \Gamma_{1}=2$. Comme $W_{k}$ n'est pas réduit, $\Gamma \simeq \mathbb{P}_{k}^{1}$ et est de multiplicité 2 dans $\left(Y_{1}\right)_{k}$. Comme $\Gamma_{1}$ est de multiplicité 1 (lemme 8 (a)), on a $\Gamma^{2}=-1$. En vertu du critère de Castelnouovo ([Chi], Theorem 3.1), $\Gamma$ est un diviseur exceptionnel. Donc $\widetilde{W} \not X$.

Supposons maintenant $g$ impair. En utilisant le corollaire 2 (a) et les lemmes 6 et 7 , on trouve que $W_{k}$ n'est pas réduit, $p_{0} \in W_{k}(k), \delta\left(p_{0}\right)=2 g+2, \lambda\left(p_{0}\right)=g+2$, $\lambda_{2}\left(p_{0}\right)=2 g+4, \lambda_{3}\left(p_{0}\right)=2 g+5$ et que $p_{0}$ est l'unique point singulier de $W$. Soit $Y_{2} \rightarrow W$ le morphisme construit dans $\S 6.3$ relatif à $p_{0}$ avec $r=2$. Alors $Y_{2}$ est régulier dans un voisinage du transformé strict de $W_{k}$ dans $Y_{2}$. On montre comme ci-dessus que $\widetilde{W} \not X$.

Soit $W_{1}=W\left(p_{0}\right)$ le modèle de Weierstrass associé à $p_{0} \in W_{k}(k)$ (voir déf. 12). Alors $W_{1}$ est minimal (lemme $\left.9(\mathrm{a})\right)$. Soit $p_{0}^{\prime} \in W_{1} \cap\left(W_{1} \wedge W\right)$. Alors $\lambda_{2}\left(p_{0}^{\prime}\right)=2 g+4$ et $W_{1}\left(p_{0}^{\prime}\right)=W$ (lemme $9(\mathrm{~b})$ ). D'après la proposition $2, W$ et $W_{1}$ sont les deux seuls modèles de Weierstrass minimaux de $C$.

Soit $Z_{1}$ le modèle de $\mathbb{P}_{K}^{1}$ défini dans $\S 6.3$ relatif à $p_{0}$ et $r=1$. Soient $Z \rightarrow Z_{1}$ l'éclatement du point d'intersection des deux composantes irréductibles de $\left(Z_{1}\right)_{k}$ et $Y \rightarrow Z$ la normalisation de $Z$ dans $C$. Alors $Z_{k}$ a exactement trois composantes irréductibles $\Theta_{0}, \Theta_{1}, \Theta_{2}$, les deux premières sont de multiplicité 1 et correspondent respectivement à $(W /\langle\sigma\rangle)_{k}$ et $\left(W_{1} /\langle\sigma\rangle\right)_{k}$, et $\Theta_{2}$ est de multiplicité 2 . Il y a une seule composante irréductible $\Gamma_{i}$ de $Y_{k}$ au-dessus de $\Theta_{i}$. De plus $Y$ est isomorphe à $Y_{2}$ dans un voisinage de $\Gamma_{0}$. Il suit que les points singuliers de $Y$ sont concentrés dans $\Gamma_{0} \backslash\left(\Gamma_{2} \cup \Gamma_{1}\right)$. Par symétrie, les points singuliers de $Y$ sont contenus dans $\left(\Gamma_{0} \cup \Gamma_{1}\right) \backslash \Gamma_{2}$. Soit $\widetilde{Y} \rightarrow Y$ la désingularisation minimale de $Y$. Comme $Y /\langle\sigma\rangle=Z$ est régulier et que $m\left(\Theta_{2}\right)=2$, toute composante irréductible de $\tilde{Y} /\langle\sigma\rangle$ au-dessus d'un point de $\Theta_{2} \backslash\left(\Theta_{0} \cup \Theta_{1}\right)$ est de multiplicité paire. Il suit que toute composante irréductible de $\widetilde{Y}_{k}$ distinctes de $\Gamma_{0}$ et $\Gamma_{1}$ est de multiplicité paire. Cela est donc vraie pour les composantes irréductibles de $X_{k}$ puisque $\Gamma_{0}$ et $\Gamma_{1}$ sont des diviseurs exceptionnels.

Remarque 15. Conservons les hypothèses de la proposition 5 . On peut montrer que si $g$ est pair, alors le morphisme canonique $\widetilde{W} \rightarrow X$ est simplement la contraction du transformé strict de $W_{k}$ dans $\widetilde{W}$. Cela est encore vrai si $g$ est impair et $\operatorname{car}(k) \neq 2$. 
Proposition 6. Soit $W$ un modèle de Weierstrass minimal de $C$ sur $\mathcal{O}_{K}$. Soient $\widetilde{W} \rightarrow W$ la désingularisation minimale de $W$ et $X$ le modèle régulier minimal de $C \operatorname{sur} \mathcal{O}_{K}$. Supposons que pour tout point $p_{0} \in W_{k}$ on ait $\lambda\left(p_{0}\right) \leq g+1$ si $g$ est pair et $\lambda_{2}\left(p_{0}\right) \leq 2 g+3$ sig est impair. Alors $\widetilde{W} \simeq X$.

Preuve. Soit $\mathcal{O}_{L}$ une extension étale de $\mathcal{O}_{K}$. D'après la prop. $4, W_{\mathcal{O}_{L}}$ est encore un modèle de Weierstrass minimal et le nouveau modèle régulier minimal est simplement $X_{\mathcal{O}_{L}}$ (lemme 11). On peut donc remplacer $\mathcal{O}_{K}$ par une extension étale maximale de sorte que $k$ soit algébriquement clos.

Procédons maintenant par l'absurde. Supposons que $\widetilde{W} \nsucceq X$. Soit $\Gamma \subset \widetilde{W}$ le transformé strict d'une composante irréductible de $W_{k}$. Alors $\Gamma$ est un diviseur exceptionnel. Soit $\Theta$ l'image de $\Gamma$ dans $Z:=\widetilde{W} /\langle\sigma\rangle$. On est dans l'une des situations suivantes :

(i) $\Theta$ rencontre une seule autre composante irréductible $\Theta^{\prime}$ de $Z_{k}$ et $\Theta^{\prime}$ est de multiplicité 1 dans $Z_{k}$;

(ii) $\Theta$ rencontre exactement deux autres composantes irréductibles de $Z_{k}$ en deux points distincts; ces composantes sont de multiplicité 1 ;

(iii) $\Theta$ rencontre une seule autre composante irréductible $\Theta^{\prime}$ qui est de multiplicité 2.

La suite de la preuve se fera en distinguant ces trois situations.

Supposons que l'on est dans la situation (i). On peut voir comme dans [Liu2], lemme 4 que le point d'intersection $\Theta \cap \Theta^{\prime}$ est d'épaisseur $e \in\{1,2\}$ (plus précisément, $e=2$ si $W_{k}$ est intègre, $e=1$ sinon). À la composante irréductible $\Theta$ (resp. $\left.\Theta^{\prime}\right)$ correspond un modèle lisse de la forme $\mathbb{P}_{x}^{1}$ (resp. $\mathbb{P}_{u}^{1}$ ), il s'obtient en contractant les composantes de $Z_{k}$ distinctes de $\Theta$ (resp. de $\Theta^{\prime}$ ). On peut supposer de plus que $u=t^{e} x$. Soient $p_{0}$ un point de $W_{k}$ correspondant à $x=0, W^{\prime}=W\left(p_{0}\right)$ (voir déf. $12)$, et $p_{0}^{\prime} \in W_{k}^{\prime} \cap\left(W^{\prime} \wedge W\right)$. Le fait que $\Gamma$ soit exceptionnel implique que $p_{0}^{\prime}$ est régulier dans $W^{\prime}$. D'après le lemme $9(\mathrm{~b})$ on a :

$$
\begin{aligned}
& \text { si } e=1, \text { alors } \lambda\left(p_{0}\right)=2 g+2-2\left[\lambda\left(p_{0}^{\prime}\right) / 2\right]=2 g+2 ; \\
& \text { si } e=2, \text { alors } \lambda^{\prime}\left(p_{0}\right)=2 g+2-2\left[\lambda\left(p_{0}^{\prime}\right) / 2\right]=2 g+2 .
\end{aligned}
$$

Donc dans les deux cas, on a $\max \left\{\lambda\left(p_{0}\right), \lambda^{\prime}\left(p_{0}\right)\right\} \geq 2 g+2$. Cela est impossible car $W$ est minimal.

Supposons que l'on est dans le cas (ii). Alors $\Gamma$ est de multiplicité 2 dans $\widetilde{W}$, il coupe les autres composantes (qui sont de multiplicité 1) en exactement deux points. Donc $W$ contient exactement deux points singuliers $p_{1}, p_{2}$ et $W_{k}$ n'est pas réduit. Cela entraine que $\delta(p)=0$ pour tout $p \neq p_{1}, p_{2}$. Il suit du lemme 6 (c) que $2 g+2=\delta\left(p_{1}\right)+\delta\left(p_{2}\right)$. D'autre part, le point d'intersection de $\Theta$ avec une autre composante de $Z_{k}$ est un point d'épaisseur 1 ([Liu2], lemme 4). Donc si l'on désigne par $\phi_{1}: Y_{1} \rightarrow W$ le morphisme construit au $\S 6.3$ relatif au point $p_{1}$ avec $r=1$, alors $Y_{1}$ est isomorphe à $\widetilde{W}$ dans un voisinage du transformé strict de $W_{k}$. Il suit que $\lambda\left(p_{1}\right)$ est pair et que $\delta\left(p_{1}\right)=\lambda\left(p_{1}\right)-1$ (lemme 8 (a) et (d)). Par conséquent $\lambda\left(p_{1}\right)+\lambda\left(p_{2}\right)=2 g+4$. Compte tenu du fait que $\lambda\left(p_{i}\right)$ est pair, on a alors $\max \left\{\lambda\left(p_{1}\right), \lambda\left(p_{2}\right)\right\} \geq 2[(g+1) / 2]+2$. Il résulte de la prop. 2 que $W$ n'est pas minimal.

Supposons que l'on est dans le cas (iii). Le diviseur $\Gamma$ est de multiplicité 2 et rencontre une unique composante (de multiplicité 2) en un seul point. Donc $W$ contient un unique point singulier $p_{0}$ et $W_{k}$ n'est pas réduit. On a $\delta\left(p_{0}\right)=2 g+2$ 
comme ci-dessus. Les composantes $\Theta$ et $\Theta^{\prime}$ se coupe transversalement en un point régulier $z_{0}$ de $Z$ ([Liu2], lemme 4). Soit $\phi_{2}: Y_{2} \rightarrow W$ le morphisme construit au $\S 6.3$ relatif à $p_{0}$ avec $r=2$. Alors $Y_{2}$ est isomorphe à $\widetilde{W}$ dans un voisinage du transformé strict de $W_{k}$. Il suit que $\lambda_{2}\left(p_{0}\right)=\delta\left(p_{0}\right)+2=2 g+4$ (lemme $8(\mathrm{~d})$ ). Or $\lambda\left(p_{0}\right) \geq \lambda_{2}\left(p_{0}\right) / 2=g+2$ (lemme $7(\mathrm{e})$ ), il suit de la prop. 3 que $g$ est impair. D'où contradiction.

Remarque 16. On a montré en même temps que si $W$ est un modèle de Weierstrass de $C$ avec $W_{k}$ réduit ( $W$ non nécessairement minimal), et si pour tout point $p_{0} \in W_{k}$ on a $\max \left\{\lambda\left(p_{0}\right), \lambda^{\prime}\left(p_{0}\right)\right\} \leq 2 g+1$, alors $\widetilde{W} \simeq X$.

Corollaire 4. Il n'existe (à isomorphisme près) qu'un nombre fini de modèles de Weierstrass minimaux de $C$.

Preuve. Supposons que $C$ admette un modèle de Weierstrass minimal $W$ qui vérifie la condition de la prop. 5. Si $g$ est pair, alors $W$ est l'unique modèle de Weierstrass minimal d'après le corollaire 2 (b) car $W$ n'a pas de point singulier rationnel sur $k$. Le cas $g$ impair résulte de la prop. 5 (b).

Dans les autres cas, pour tout modèle de Weierstrass minimal, on a $X \simeq \widetilde{W}$. Chaque modèle minimal $W$ donne donc naissance à une ou deux composantes irréductibles de $X_{k}$ (il s'agit des transformés stricts par $X \simeq \widetilde{W} \rightarrow W$ des composantes irréductibles de $\left.W_{k}\right)$. Ces composantes irréductibles sont distinctes pour des modèles $W$ non isomorphes. Le corollaire résulte de la finitude des composantes irréductibles de $X_{k}$.

Remarque 17. Si le corps résiduel $k$ est fini, alors pour tout entier $d$, il n'existe qu'un nombre fini de modèles de Weierstrass $W$ de $C$ de discriminant $\nu\left(\Delta_{W}\right)=d$. Mais cela est faux dès que $k$ est infini.

Définition 14. Soit $Y$ un modèle de $C$ sur $\mathcal{O}_{K}$ (voir $\S 4$ ), soit $P_{0} \in C(K)$. Comme $Y$ est propre sur $\mathcal{O}_{K}$, la section $P_{0}:$ Spec $K \rightarrow C$ s'étend en une section $\operatorname{Spec} \mathcal{O}_{K} \rightarrow$ $Y$. On appelle spécialisation de $P_{0}$ dans $Y_{k}$ l'image par cette section du point fermé de $\operatorname{Spec} \mathcal{O}_{K}$ dans $Y_{k}$. C'est aussi le point d'intersection $\overline{\left\{P_{0}\right\}} \cap Y_{k}$. Si ce point est régulier dans $Y$, il est alors lisse sur $k$.

Corollaire 5. Soit $X$ le modèle régulier minimal de $C$ sur $\mathcal{O}_{K}$. Supposons $C(K) \neq$ $\emptyset$. Alors on a les propriétés suivantes:

(a) Pour tout modèle de Weierstrass minimal $W$ de $C$, on a $\widetilde{W} \simeq X$.

(b) Pour tout point $P_{0} \in C(K)$, il existe un unique modèle de Weierstrass $W$ (non nécessairement minimal) tel que la spécialisation de $P_{0}$ dans $W_{k}$ soit un point lisse et que $X \simeq \widetilde{W}$.

Preuve. (a) Supposons que $\widetilde{W} \not X$. Comme $X_{k}$ contient une composante irréductible de multiplicité 1 , il suit des prop. 5 et 6 que $g$ est pair et que $W_{k}$ n'est pas réduit. Soient $P_{0} \in C(K)$ et $p_{0}$ sa spécialisation dans $W_{k}$. Alors $p_{0}$ est un point singulier de $W$ rationnel sur $k$. Or on a vu dans la preuve de la prop. 5 que $W$ contient un unique point singulier et qu'il n'est pas rationnel sur $k$, d'où contradiction.

(b) Soit $\Gamma$ une composante irréductible de $X_{k}$ contenant la spécialisation de $P_{0}$ dans $X_{k}$. Alors $\Gamma$ est de multiplicité 1 dans $X_{k}$. Soit $\Theta$ l'image de $\Gamma$ dans $X /\langle\sigma\rangle$, alors il existe un unique modèle $\mathbb{P}_{x}^{1}$ de $\mathbb{P}_{K}^{1}$ birationnel à $X /\langle\sigma\rangle$ dans un voisinage 
du point générique de $\Theta$. La normalisation de $\mathbb{P}_{x}^{1}$ dans $C$ est l'unique modèle de Weierstrass répondant à la question.

\section{Discriminant et multiplicité}

Soit $W$ un modèle de Weierstrass de $C$ sur $\mathcal{O}_{K}$. Dans ce paragraphe, on donne une majoration des multiplicités $\lambda\left(p_{0}\right)$ par le discriminant $\nu\left(\Delta_{W}\right)$ (prop. 7). On en déduit notamment que si $\nu\left(\Delta_{W}\right)=1$, alors $W$ est régulier. On peut améliorer ces résultats lorsque $\operatorname{car}(k) \neq 2(\S 9.2)$ grâce à la notion du discriminant local.

En fin du paragraphe, on décrit la désingularisation minimale de $W$ en $p_{0}$ lorsque $\delta\left(p_{0}\right) \leq 2$. Cette condition implique que $p_{0}$ est une singularité de type $\mathrm{A}_{n}$ ou $\mathrm{D}_{n}$ (dans la classification des singularités rationnelles). La majoration ci-dessus implique que $n \leq \nu\left(\Delta_{W}\right)$.

9.1 Une majoration par le discriminant.

Considérons l'anneau des polynômes $\mathbb{Z}\left[u_{i}, v_{j}\right]_{0 \leq i \leq g+1}, 0 \leq j \leq 2 g+2$. Soit $\Delta$ le discriminant de l'équation hyperelliptique

$$
y^{2}+\left(u_{g+1} x^{g+1}+\cdots+u_{1} x+u_{0}\right) y=v_{2 g+2} x^{2 g+2}+\cdots+v_{1} x+v_{0}
$$

On sait que $\Delta \in \mathbb{Z}\left[u_{i}, v_{j}\right]_{i, j}$. Le lemme suivant précise un peu plus sa nature.

Lemme 12. Soit $1 \leq n \leq 2 g+2$, soit $I_{n}$ l'idéal $\left(v_{i}, u_{j} u_{l}\right)_{0 \leq i \leq n, j+l \leq n}$ de $\mathbb{Z}\left[u_{i}, v_{j}\right]_{i, j}$. Alors on a

$$
\Delta \in\left(I_{n}\right)^{n} \cap\left(v_{0}, u_{0}^{2}, v_{1}^{2}, u_{0} u_{1} v_{1}\right) \cap\left(v_{i}, u_{j} u_{l}\right)_{i, j, l}^{4 g+2} .
$$

Preuve. Montrons que $\Delta \in\left(I_{n}\right)^{n}$. l'appartenance aux deux autres idéaux se démontrent de façon analogue. Il est clair que $\mathbb{Z}\left[u_{i}, v_{j}\right] /\left(I_{n}\right)^{n}$ est sans torsion sur $\mathbb{Z}$. Il suffit donc de montrer l'assertion dans $\mathbb{Q}\left[u_{i}, v_{j}\right]$. Soit

$$
a_{i}=4 v_{i}+\sum_{j+l=i} u_{j} u_{l} .
$$

Alors $\Delta$ est le discriminant de $\sum a_{i} x^{i}$ (à une puissance entière de 2 près), c'est aussi le discriminant $\operatorname{disc}(\mathcal{P}(x))$ de $\mathcal{P}(x):=\sum a_{i} x^{2 g+2-i}$. Soit $J_{n}$ l'idéal $\left(a_{0}, a_{1}, \ldots, a_{n}\right)$ de $\mathbb{Q}\left[a_{i}\right]_{0 \leq i \leq 2 g+2}$, il suffit de montrer que $\Delta \in\left(J_{n}\right)^{n}$. Or

$$
\operatorname{disc}(\mathcal{P}(x))=a_{0}^{-1} \operatorname{Res}\left(\mathcal{P}(x), \mathcal{P}^{\prime}(x)\right) .
$$

La propriété se voit alors en développant par rapport aux $n+1$ premières lignes du déterminant (qui donne le résultant) :

$$
\left|\begin{array}{cccccccc}
(2 g+2) a_{0} & 0 & 0 & \ldots & a_{0} & 0 & 0 & \ldots \\
(2 g+1) a_{1} & (2 g+2) a_{0} & 0 & \ldots & a_{1} & a_{0} & 0 & \ldots \\
\vdots & \vdots & \vdots & \vdots & \vdots & \vdots & \vdots & \vdots
\end{array}\right|
$$

Soit $p_{0} \in W_{k}$ un point rationnel sur $k$, soit $W^{\prime}$ un modèle de Weierstrass non isomorphe à $W$ tel que $p_{0} \in W^{\prime} \wedge W$ (déf. 7). Considérons la suite de modèles de Weierstrass $W^{0}=W, W^{1}, \ldots, W^{d}=W^{\prime}$ construit dans la preuve de la proposition 3 , où $d=d\left(W, W^{\prime}\right)$. Notons $p_{i}$ un point de $W^{i} \cap\left(W^{i} \wedge W^{i+1}\right)$. Ce point est unique s'il est singulier dans $W^{i}$. Posons

$$
\lambda\left(p_{0}, p_{d-1}\right):=\lambda\left(p_{d-1}\right)+\sum_{0 \leq i \leq d-2} 2\left[\lambda\left(p_{i}\right) / 2\right] .
$$

Notons $\epsilon=0$ si $W_{k}$ est réduit et $\epsilon=1$ sinon. 
Lemme 13. Avec les notations ci-dessus on a

$$
\min \left\{\lambda\left(p_{0}, p_{d-1}\right)+(4 g+1) \epsilon, 2 \lambda\left(p_{0}, p_{d-1}\right)-2 d+4 g \epsilon\right\} \leq \nu\left(\Delta_{W}\right) .
$$

En particulier, si les $p_{i}$ sont singuliers dans $W^{i}$, alors $2 d \leq \nu\left(\Delta_{W}\right)$.

Preuve. On peut supposer que $W /\langle\sigma\rangle=\mathbb{P}_{x}^{1}, W^{\prime} /\langle\sigma\rangle=\mathbb{P}_{u}^{1}$ et $x=t^{d} u$. Pour tout $i \leq d$, notons $B^{i}$ la clôture intégrale de $\mathcal{O}_{K}\left[x / t^{i-1}\right]$ dans $K(C)$ et

$$
\lambda^{i}\left(\sum_{j} c_{j} x^{j}\right)=\min \left\{\nu\left(c_{j}\right)+i j \mid j \geq 0\right\} .
$$

Pour toute base $\{1, y\}$ de $B:=B^{1}$, on note $\lambda^{i}(y)=\min \left\{2 \lambda^{i}(Q(x)), \lambda^{i}(P(x))\right\}$ et $\lambda^{i}$ le maximum des $\lambda^{i}(y)$. On montre comme pour le lemme 7 (c) qu'il existe une base standard $\{1, y\}$ de $B$ telle que $\lambda^{i}(y)=\lambda^{i}$ pour tout $1 \leq i \leq d$, et que si $W_{k}$ n'est pas réduit alors $\widetilde{Q}(x)=\widetilde{P}(x)=0$. Il est alors clair que $\lambda^{i}=\lambda\left(p_{i-1}\right)+2\left[\lambda^{i-1} / 2\right]$ pour tout $i \geq 2$. Donc $\lambda^{d}=\lambda\left(p_{0}, p_{d-1}\right)$.

D'autre part, si on écrit $Q(x)=\sum_{i} b_{j} x^{j}$ et $P(x)=\sum_{j} a_{j} x^{j}$ (pour une base standard $\{1, y\}$ choisie comme ci-dessus), alors $2 \nu\left(b_{j}\right), \nu\left(a_{j}\right) \geq \lambda^{d}-j d$ par définition. Or d'après le lemme 12 , le discriminant de l'équation $y^{2}+Q(x) y=P(x)$ appartient à $\left(a_{0}, a_{1}^{2}, b_{0}^{2}, a_{1} b_{0} b_{1}\right) \mathbb{Z}\left[a_{i}, b_{j}\right]$. De plus, si l'on affecte les $a_{i}$ de degré 1 et les $b_{j}$ de degré $1 / 2$, alors $\Delta$ est homogène de degré $4 g+2$. Cela donne l'inégalité

$$
\nu\left(\Delta_{W}\right) \geq \min \left\{\lambda^{d}+(4 g+1) \epsilon, 2\left(\lambda^{d}-d\right)+4 g \epsilon\right\} .
$$

D'où le lemme.

Proposition 7. Soient $W$ un modèle de Weierstrass de $C$ sur $\mathcal{O}_{K}$ et $p_{0}$ un point singulier de $W$. Posons $\epsilon=0$ si $W_{k}$ est réduit et $\epsilon=1$ sinon.

(a) Si $\nu\left(\Delta_{W}\right) \leq 4 g+1$, alors $W_{k}$ est réduit, et $W$ est l'unique modèle de Weierstrass minimal de $C$.

(b) On a $\nu\left(\Delta_{W}\right) \geq \lambda\left(p_{0}\right)+4 g \epsilon$, et $\nu\left(\Delta_{W}\right) \geq \delta\left(p_{0}\right)-1$.

Preuve. (a) Le fait que $W_{k}$ soit réduit est une conséquence immédiate du lemme 12. De plus, pour tout modèle de Weierstrass $W^{\prime}$ de $C$, la différence $\nu\left(\Delta_{W^{\prime}}\right)-\nu\left(\Delta_{W}\right)$ est un multiple de $4 g+2$ (voir $\S 2$ ). D'où le résultat.

(b) Quitte à faire un changement de base étale (voir §8), on peut supposer que $p_{0}$ est rationnel sur $k$. Alors la première inégalité résulte du lemme 13 en prenant $W^{\prime}=W\left(p_{0}\right)$ (déf. 12$)$, et donc $d=d\left(W^{\prime}, W\right)=1, \lambda\left(p_{0}, p_{d-1}\right)=\lambda\left(p_{0}\right)$.

Soit $y^{2}+Q(x) y=P(x)$ une équation entière associée à $W$ telle que $x\left(p_{0}\right)=0$, $\lambda\left(p_{0}, y\right)=\lambda\left(p_{0}\right), \delta\left(p_{0}, y\right)=\delta\left(p_{0}\right)$. Ecrivons $Q(x)=\sum_{i} b_{i} x^{i}, P(x)=\sum_{i} a_{i} x^{i}$. Alors par définition $\nu\left(a_{i}\right) \geq 1$ si $i \leq \delta\left(p_{0}\right)-1$ et $\nu\left(b_{j}\right) \geq 1$ si $j \leq \delta\left(p_{0}\right) / 2-1$. Donc d'après le lemme 12 (avec $\left.n=\delta\left(p_{0}\right)-1\right)$, on a $\nu\left(\Delta_{W}\right) \geq \delta\left(p_{0}\right)-1$.

Corollaire 6. Soit $W$ un modèle de Weierstrass de $C$. Si $\nu\left(\Delta_{W}\right)=1$, alors $W$ est régulier.

Preuve. C'est une conséquence directe de la prop. 7 (b).

Remarque 18. Gardons l'hypothèse du corollaire 6 . Alors $W_{k}$ est réduit et n'admet que des points doubles ordinaires comme singularité en vertu de la prop. 7 . Si $\operatorname{car}(k) \neq 2$, on voit en utilisant le corollaire 7 ci-après que $W_{k}$ est intègre et que $W_{k}$ n'admet qu'un point singulier qui est de plus rationnel sur $k$. 
Cela est encore vrai si $\operatorname{car}(k)=2$ et si $C$ est elliptique ou de genre $g=2$ (utiliser la formule d'Ogg si $C$ est elliptique et [Liu2], théorèmes 1 et 2 si $g=2$ ). Si $g \geq 3$, cet énoncé est certainement valable encore, mais nécessiterait une étude plus poussée.

\subsection{Facteurs locaux du discriminant.}

On suppose dans cette section que $\operatorname{car}(k) \neq 2$. Soit $W$ un modèle de Weierstrass de $C$. A chaque point $p \in W_{k}$, on va associer un discriminant $\nu\left(\Delta_{W, p}\right)$ de sorte que l'on retrouve $\nu\left(\Delta_{W}\right)$ par une sommation sur les $\nu\left(\Delta_{W, p}\right)$. Soit $p_{0} \in W_{k}$ tel que $\delta\left(p_{0}\right) \geq 1$. Soit $x \in K(C)^{\langle\sigma\rangle}$ tel que $W /\langle\sigma\rangle=\mathbb{P}_{x}^{1}$ et que $x$ ne soit pas un pôle de $p_{0}$. On peut donc écrire une équation affine $y^{2}=P(x)$ de $W$. Soit $\epsilon$ comme dans la prop. 7. Posons $H(x)=t^{-\epsilon} P(x)$. On peut décomposer l'image $\widetilde{H}(x)$ de $H(x)$ dans $k[x]: \widetilde{H}(x)=s_{0}(x)^{\delta\left(p_{0}\right)} r(x)$ avec $s_{0}(x)$ unitaire, irréductible et s'annulant en $p_{0}, r(x)$ premier à $s_{0}(x)$. Soit $\mathcal{O}_{K}^{h}$ l'hensélisé de $\mathcal{O}_{K}$. Alors on a une unique décomposition dans $\mathcal{O}_{K}^{h}: H(x)=H_{0}(x) R(x)$, avec $H_{0}(x), R(x) \in \mathcal{O}_{K}^{h}[x], H_{0}(x)$ unitaire, $\widetilde{H}_{0}(x)=s_{0}(x)^{\delta\left(p_{0}\right)}$ et $\widetilde{R}(x)=r(x)$.

On note encore par $\nu$ l'unique prolongement de $\nu$ sur l'hensélisé $K^{h}$ de $K$.

Définition 15. Avec les notations ci-dessus, on appelle facteur local du discriminant $\nu\left(\Delta_{W}\right)$ en $p_{0}$ la valuation du discriminant de l'équation hyperelliptique $y_{0}^{2}=$ $t^{\epsilon} H_{0}(x)$ (voir $\left.\S 2\right)$. On le note $\nu\left(\Delta_{W, p_{0}}\right)$.

On note $C_{p_{0}}$ la courbe hyperelliptique sur $K^{h}$ définie par l'équation $y_{0}^{2}=t^{\epsilon} H_{0}(x)$, et $W_{p_{0}}$ le modèle de Weierstrass de $C_{p_{0}}$ sur $\mathcal{O}_{K}^{h}$ associé à cette équation.

Lemme 14. Avec les notations ci-dessus, on a les propriétés suivantes :

(a) Il existe un unique point singulier $q_{0}$ dans $W^{\prime}:=W_{p_{0}}$, et après une extension étale de $\mathcal{O}_{K}$, on a un isomorphisme des complétés formels relatifs aux idéaux maximaux

$$
\widehat{\mathcal{O}}_{W, p_{0}} \simeq \widehat{\mathcal{O}}_{W^{\prime}, q_{0}} .
$$

(b) On a $\delta\left(q_{0}\right)=\delta\left(p_{0}\right)$ et $\lambda\left(q_{0}\right)=\lambda\left(p_{0}\right)$.

(c) Soit $\epsilon$ comme dans la prop. \%. On a

$$
\nu\left(\Delta_{W}\right)-(4 g+2) \epsilon=\sum_{\delta(p) \geq 1}\left(\nu\left(\Delta_{W, p}\right)-(4[(\delta(p)-1) / 2]+2) \epsilon\right)[k(p): k] .
$$

Preuve. (a) Soit $q_{0}$ le point de $W^{\prime}$ correspondant à $s_{0}(x)=t=0$. Alors par construction, $\delta\left(q_{0}\right)=\delta\left(p_{0}\right)=\left(\operatorname{deg} \widetilde{H}_{0}(x)\right) /\left[k\left(p_{0}\right): k\right]$. C'est donc l'unique point singulier de $W^{\prime}$. D'autre part, après extension étale de $\mathcal{O}_{K}$, l'image dans $k[x] \mathrm{du}$ polynôme noté $R(x)$ plus haut est le carré d'un élément non nul, donc $R(x)$ est un carré dans le complété formel $\widehat{\mathcal{O}}_{W, p_{0}}$, ce qui implique l'isomorphisme souhaité.

(b) L'entier $\lambda\left(p_{0}\right)$ est la multiplicité en $p_{0}$ du diviseur de ramification de $W \rightarrow$ $W /\langle\sigma\rangle$, il ne dépend donc que de l'anneau local complété $\widehat{\mathcal{O}}_{W, p_{0}}$. D'où $\lambda\left(p_{0}\right)=$ $\lambda\left(q_{0}\right)$. Cela peut se voir directement par la définition aussi.

(c) Quitte à faire un changement de base étale, on peut choisir $x \in K(C)^{\langle\sigma\rangle}$ de sorte que $W /\langle\sigma\rangle=\mathbb{P}_{x}^{1}$ et que le diviseur des pôles de $x$ soit contenu dans le lieu régulier de $W_{k}$. Soit $y^{2}=P(x)$ l'équation de $W$ associé à $x$. L'égalité sur $\nu\left(\Delta_{W}\right)$ résulte de l'expression du discriminant en fonction des différences des zéros de $P(x)$ dans une clôture algébrique de $K$. Notons que par définition $\nu\left(\Delta_{W, p_{0}}\right)=$ $\nu\left(\operatorname{disc}\left(H_{0}\right)\right)+\left(4\left[\left(\delta\left(p_{0}\right)-1\right) / 2\right]+2\right) \epsilon$, et que les deux membres de l'égalité à démontrer ne changent pas après extension étale de $\mathcal{O}_{K}$. 
Corollaire 7. Supposons $\operatorname{car}(k) \neq 2$. Soit $W$ un modèle de Weierstrass de $C$ tel que $W_{k}$ soit réduit. Alors on a

$$
\nu\left(\Delta_{W}\right) \geq \sum_{p \in W_{k}, \lambda(p) \geq 2} \lambda(p)[k(p): k] .
$$

Preuve. C'est une conséquence immédiate du lemme 14 (c) et de la prop. 7 (b).

Remarque 19. Nous ne savons pas si la construction du facteur local $\Delta_{W, p}$ peut encore se faire lorsque $\operatorname{car}(k)=2$. Cependant, l'inégalité ci-dessus peut être encore valable.

9.3 La désingularisation de $W$ dans quelques cas simples.

Soit $W$ un modèle de Weierstrass de $C$ et $p_{0} \in W_{k}$ un point singulier de $W$. Nous décrivons dans ce qui suit le processus de désingularisation de $W$ en $p_{0}$ dans quelques cas simples. On suppose $p_{0}$ rationnel sur $k$ pour simplifier.

(9.3.1) Supposons $\delta\left(p_{0}\right)=1$. Alors $W_{k}$ est non réduit et $\lambda\left(p_{0}\right)=2$. De plus, $\lambda_{2}\left(p_{0}\right)=3, \lambda^{\prime}\left(p_{0}\right) \leq 1$ (lemme 7 (e), lemme 9 (c)). Donc l'éclatement $Y_{1} \rightarrow W$ de centre $p_{0}$ désingularise $p_{0}$, l'image réciproque $\Gamma$ de $p_{0}$ dans $Y_{1}$ est isomorphe à $\mathbb{P}_{k}^{1}$ et est de multiplicité 1 . De plus $\Gamma$ intersecte le transformé strict $\widetilde{W}_{k}$ transversalement (utiliser la formule de projection comme dans la preuve de la prop. 5).

(9.3.2) Supposons $\delta\left(p_{0}\right)=2$ et $W_{k}$ réduit. Alors $p_{0}$ est un point double ordinaire. L'image réciproque de $p_{0}$ par $\widetilde{W} \rightarrow W$ est donc une chaîne de $n$ droites projectives sur $k$. Le point $p_{0}$ est une singularité de type $\mathrm{A}_{n}$ dans $W$. L'entier $n+1$ est l'épaisseur du point double ordinaire $p_{0}$ dans $W$. On peut déterminer $n$ par l'algorithme qui suit.

Comme $p_{0}$ est singulier par hypothèse, on a $\lambda\left(p_{0}\right)=2$ d'après le lemme 7 (e). Soit $W^{1}=W\left(p_{0}\right)$ le modèle de Weierstrass associé au point $p_{0}$ (voir déf. 12). Alors $W_{k}^{1}$ est réduit, et d'après le lemme $9(\mathrm{~d}), W^{1}$ admet au plus un point singulier $p_{1}$ qui est alors rationnel sur $k$ et on a $\delta\left(p_{1}\right)=2$. Si $\lambda\left(p_{1}\right) \leq 1$, on pose $m=1$ et l'algorithme est fini. Sinon, on considère $W^{2}:=W\left(p_{1}\right)$ et on continue ainsi de suite. Lorsque $\lambda\left(p_{m}\right) \leq 1$, l'algorithme s'arrête. Si $W_{k}^{m}$ est irréductible, alors $n=2 m-1$, sinon $n=2 m$.

D'après le lemme 13 (appliqué à la suite $\left.W, W^{1}, \ldots, W^{m}\right)$, on a $n \leq \nu\left(\Delta_{W}\right)$ car $\lambda\left(p_{0}, p_{m-1}\right)=2 m$.

(9.3.3) Supposons $\delta\left(p_{0}\right)=2$ et $W_{k}$ est non réduit. Alors $\lambda\left(p_{0}\right) \leq 3$.

Si $\lambda\left(p_{0}\right)=2$, alors $\lambda^{\prime}\left(p_{0}\right) \leq 1$. On peut résoudre "à la main" la singularité $p_{0}$. On trouve la figure 1 .

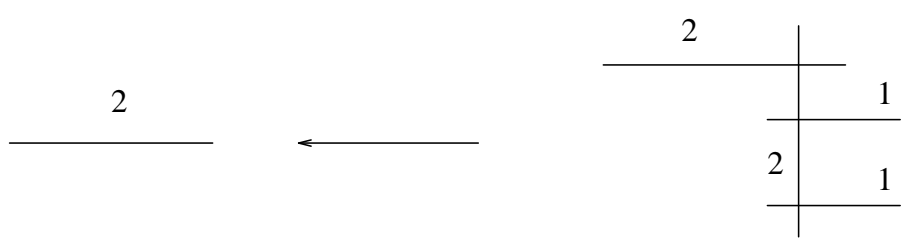

FiguRe 1

Les composantes irréductibles de l'image réciproque de $p_{0}$ sont des coniques lisses sur $k$. 


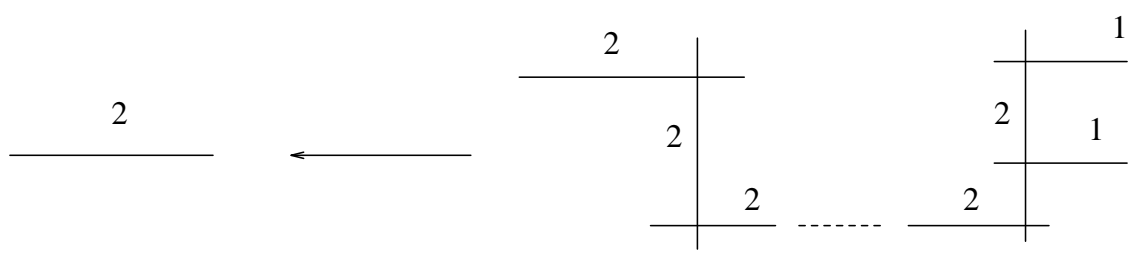

FiguRE 2

Supposons maintenant $\lambda\left(p_{0}\right)=3$. On va construire une certaine suite de modèles de Weierstrass $W, W^{1}, \ldots, W^{m}$. Posons $W^{1}=W\left(p_{0}\right)$ (déf. 12). Alors $W_{k}^{1}$ n'est pas réduit, $\lambda^{\prime}\left(p_{0}\right) \leq 3$ et on a

$$
\sum_{p \in W_{k}^{1} \backslash\left(W^{1} \wedge W\right)} \delta(p)[k(p): k]=2
$$

(lemme 9). Si $\lambda^{\prime}\left(p_{0}\right) \leq 2$, on s'arrête. Sinon, soit $p_{1}$ l'unique point de $W_{k}^{1} \backslash\left(W^{1} \wedge W\right)$ tel que $\lambda\left(p_{1}\right)=3$. Alors $\delta\left(p_{1}\right)=2, p_{1} \in W_{k}^{1}(k)$. On continue alors la construction. On s'arrête à $W^{m}=W^{m-1}\left(p_{m-1}\right)$ lorsque $\lambda^{\prime}\left(p_{m}\right)=2$ (et $\left.\lambda\left(p_{m}\right)=3\right)$. Cette suite s'arrête nécessairement car le lemme 13 donne la majoration $2 m+3 \leq \nu\left(\Delta_{W}\right)$. Soient $W^{m+1}=W^{m}\left(p_{m}\right), \delta^{\prime}\left(p_{m}\right)=\max \left\{\delta(p) \mid p \in W_{k}^{m+1}, p \notin W^{m} \wedge W^{m+1}\right\}$.

Un raisonnement analogue à ceux qui précèdent montre que la désingularisation minimale $\phi: \widetilde{W} \rightarrow W$ de $W$ en $p_{0}$ est de la forme figure 2 (après éventuellement une extension quadratique de $k$ ) où les composantes irréductibles de $\phi^{-1}\left(p_{0}\right)$ sont des $\mathbb{P}^{1}$ (sur une clôture algébrique $\bar{k}$ de $k$ ), leur nombre $n$ (sur $\bar{k}$ ) est déterminé par $n=2 m+3$ si $\delta^{\prime}\left(p_{m}\right)=1$ et $n=2 m+4$ sinon. On a ici une singularité de type $\mathrm{D}_{n}$, avec $n \leq \nu\left(\Delta_{W}\right)$. Les discussions ci-dessus permettent d'énoncer le corollaire suivant :

Corollaire 8. Soit $W$ un modèle de Weierstrass de $C$, soit $p_{0}$ un point de $W_{k}(k)$ tel que $\delta\left(p_{0}\right) \leq 2$. Alors $p_{0}$ est une singularité rationnelle de type $A_{n}$ ou $D_{n}$, avec $n \leq \nu\left(\Delta_{W}\right)$.

\section{Applichtions aux courbes elliptiques}

On s'intéresse dans ce paragraphe au cas des courbes elliptiques, et plus particulièrement à l'algorithme de Tate qui détermine la réduction du modèle régulier minimal. Les résultats sont essentiellement bien connus. Mais nos démonstrations permettent d'illustrer la théorie générale développée dans les paragraphes précédents.

On suppose dans tout ce paragraphe que $g=1, C(K) \neq \emptyset$. On fixera un point $o \in C(K)$ et une involution hyperelliptique $\sigma$ de $C$ telle que $\sigma(o)=o$. Sauf mention expresse du contraire, les modèles de Weierstrass considérés sont relatifs à $\sigma$. Lorsque l'on considère $C$ comme une courbe elliptique, l'élément neutre sera sous-entendu $o$. On note $X$ le modèle régulier minimal de $C$ sur $\mathcal{O}_{K}$.

\subsection{Modèles de Weierstrass minimaux.}

Considérons le modèle de Weierstrass minimal $W_{0}$ de $(C, o)$ sur $\mathcal{O}_{K}$. Par définition, c'est un modèle de Weierstrass de $C \operatorname{sur} \mathcal{O}_{K}$ tel que la spécialisation de $o$ dans $\left(W_{0}\right)_{k}$ soit un point lisse sur $k$ et que $\nu\left(\Delta_{W}\right)$ soit minimal pour cette propriété. Il est bien connu que ce modèle est unique à isomorphisme près. 
Le fait que la spécialisation de $o$ dans $\left(W_{0}\right)_{k}$ soit lisse peut se traduire de la façon suivante : $W_{0}$ est associé à une équation entière (déf. 2 )

$$
y^{2}+\left(a_{1} x+a_{3}\right) y=x^{3}+a_{2} x^{2}+a_{4} x+a_{6}
$$

et $o$ est le pôle de $x$.

Définition 16. Soit $W$ un modèle de Weierstrass de $(C, \sigma)$ sur $\mathcal{O}_{K}$. Soit

$$
y^{2}+Q(x) y=P(x)
$$

une équation entière de $C$ associée à $W$. On appelle différentielle canonique de $W$ la forme différentielle

$$
\omega(W):=d x /(2 y+Q(x)) .
$$

On vérifie aisément que modulo $\mathcal{O}_{K}^{*}, \omega(W)$ est indépendant du choix de $x$ et de $y$.

Soit $\omega_{W / \mathcal{O}_{K}}$ le faisceau dualisant relatif de $W \operatorname{sur} \mathcal{O}_{K}$. On a canoniquement $\omega_{W / \mathcal{O}_{K}}=\omega(W) \mathcal{O}_{W}$ (utiliser par exemple [Liu2], lemme 2).

Proposition 8. Soit $(C, o)$ une courbe elliptique sur $K$. Soient $X$ son modèle régulier minimal sur $\mathcal{O}_{K}$ et $W$ un modèle de Weierstrass de $C$ sur $\mathcal{O}_{K}$. On a les propriétés suivantes :

(a) Soit $\widetilde{W} \rightarrow W$ la désingularisation minimale de $W$. Si $\widetilde{W} \simeq X$, alors le faisceau dualisant relatif $\omega_{X / \mathcal{O}_{K}}$ vérifie $\omega_{X / \mathcal{O}_{K}}=\omega(W) \mathcal{O}_{X}$. En particulier, $\mathrm{H}^{0}\left(X, \omega_{X}\right)=\omega(W) \mathcal{O}_{K}$.

(b) On a $\widetilde{W} \simeq X$ si et seulement si $W$ est minimal.

(c) Le modèle de Weierstrass minimal $W_{0}$ de $(C, o)$ est un modèle de Weierstrass minimal de $C$ en tant que courbe hyperelliptique. En particulier, le discriminant minimal de la courbe elliptique $(C, o)$ coïncide avec le discriminant minimal de $(C, \sigma)$.

Preuve. (a) Soit $\pi: X \rightarrow W$ le morphisme canonique. Par la théorie d'intersection sur $X$, pour toute composante irréductible $\Gamma$ de $X_{k}$, distincte du transformé strict de $W_{k}$, on a $\omega_{X} \cdot \Gamma=0$. Il suit que $\omega_{X}=\pi^{*} \omega_{W}=\omega(W) \mathcal{O}_{X}$ ([Lip], Theorem 27.1) et donc $\mathrm{H}^{0}\left(X, \omega_{X}\right)=\omega(W) \mathcal{O}_{K}$.

(b) Si $W$ est minimal, on a $\widetilde{W} \simeq X$ en vertu du corollaire 5 (a). Inversement supposons que $\widetilde{W} \simeq X$. Soit $\left(\mathcal{E}^{\prime}\right)($ resp. $(\mathcal{E})$ ) une équation entière associée à un modèle de Weierstrass minimal $W^{\prime}$ de $C$ (resp. à $W$ ). En vertu de (a), on a $\omega(W) \mathcal{O}_{K}^{*}=\omega\left(W^{\prime}\right) \mathcal{O}_{K}^{*}$. Or on peut vérifier à l'aide de la formule en fin du $\S 2$ que

$$
\Delta\left(\mathcal{E}^{\prime}\right) \cdot \omega\left(W^{\prime}\right)^{\otimes 12}=\Delta(\mathcal{E}) \cdot \omega(W)^{\otimes 12} \in \mathrm{H}^{0}\left(C, \Omega_{C / K}^{1}\right)^{\otimes 12} .
$$

Par conséquent, $\nu\left(\Delta_{W}\right)=\nu\left(\Delta_{W^{\prime}}\right)$ et $W$ est minimal.

(c) Soit $W$ l'unique modèle de Weierstrass de $C$ tel que $\widetilde{W} \simeq X$ et que la spécialisation de $o$ dans $W_{k}$ soit lisse (corollaire $5(\mathrm{~b})$ ). D'après (b), $W$ est minimal. Donc $W \simeq W_{0}$.

Remarque 20. La désingularisation minimale $\widetilde{W}_{0}$ de $W_{0}$ est isomorphe à $X$ d'après la prop. ci-dessus (b) et (c). Cela résulte aussi implicitement de l'algorithme de Tate ([Sil], IV.9.1).

Remarque 21. Soient $\tau$ une involution hyperelliptique de $C$ et $W_{\tau}$ un modèle de Weierstrass minimal de $(C, \tau)$. La même preuve que ci-dessus montre que l'on a 


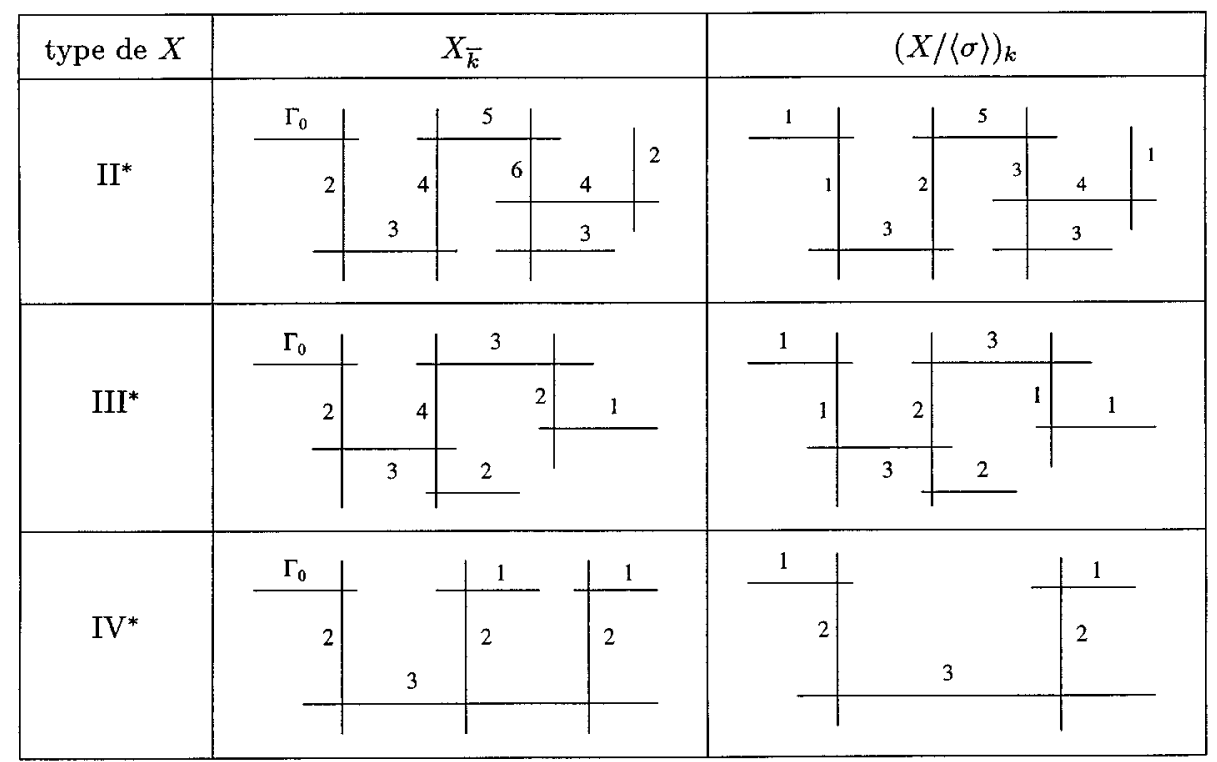

TABleau 1

$\mathrm{H}^{0}\left(X, \omega_{X}\right)=\omega\left(W_{\tau}\right) \mathcal{O}_{K}$. Donc $\nu\left(\Delta_{W_{\tau}}\right)=\nu\left(\Delta_{W_{\sigma}}\right)$. On voit donc que le discriminant minimal est indépendant du choix d'une involution hyperelliptique lorsque $C(K) \neq \emptyset$, ou mieux si $C$ a un point rationnel dans le hensélisé strict $K^{\text {sh }}$ de $K$ (prop. 4).

Prenons pour un instant une courbe hyperelliptique $C$ de genre 1 telle que $C\left(K^{\mathrm{sh}}\right)=\emptyset$. Soient $\tau$ une involution hyperelliptique de $C$ et $W_{\tau}$ un modèle de Weierstrass minimal de $C$ relatif à $\tau$. Alors $\mathrm{H}^{0}\left(X, \omega_{X}\right) \neq \omega\left(W_{\tau}\right) \mathcal{O}_{K}$. Si car $(k) \neq 2$, en utilisant la remarque 15 on voit que $t^{-1} \omega\left(W_{\tau}\right)$ est une base de $\mathrm{H}^{0}\left(X, \omega_{X}\right)$. On a donc encore l'indépendance du discriminant minimal vis-à-vis du choix d'une involution hyperelliptique. Par contre, si $\operatorname{car}(k)=2$, nous ne savons pas si ce résultat subsiste.

10.2 Action de l'involution sur le modèle régulier minimal.

Soit $(C, o)$ une courbe elliptique. Rappelons que l'involution $\sigma$ laisse invariant $o$ par hypothèse. Soit $X$ le modèle régulier minimal de $C \operatorname{sur} \mathcal{O}_{K}$. On souhaite étudier le schéma quotient $X /\langle\sigma\rangle$. D'après le corollaire 5 , il y a bijection entre les modèles de Weierstrass minimaux de $C$ et les composantes de multiplicité 1 de $(X /\langle\sigma\rangle)_{k}$.

Les cas qui nous intéressent dans la suite sont les types $\mathrm{II}^{*}$, III* et IV*. En utilisant [Liu2], lemme 4 , on obtient le tableau 1 où $\Gamma_{0}$ est la composante irréductible de $X_{k}$ qui contient la spécialisation de l'élément neutre $o$.

On note d'après le tableau que si $X$ est de type III*, alors $C$ admet exactement quatre modèles de Weierstrass minimaux. S'il est de type $\mathrm{II}^{*}$ ou $\mathrm{IV}^{*}$, alors $C$ admet exactement trois modèles de Weierstrass minimaux. De plus le type II* est caractérisé par le fait que deux de ces modèle de Weierstrass sont à fibre spéciale non réduite. 
10.3 Algorithme de Tate.

Dans cette section on souhaite déterminer le type de Kodaira-Néron de la fibre spéciale $X_{k}$. Plus précisément, soit $W$ le modèle de Weierstrass minimal de $(C, o)$, on va examiner le processus de désingularisation $X \rightarrow W$ connu sous le nom de l'algorithme de Tate ([Tat], §6-8). Cette désingularisation avait été décrite en détail par Néron ([Nér], chap. III, §10-15). La preuve complète de [Tat] se trouve aussi dans [Sil], IV.9.

La théorie d'intersection sur $X$ permet de trouver a priori toutes les configurations possibles de $X_{k}$ ([Des], $\S 4.7,[\mathrm{Kod}]$, Theorem 6.2 , ou [Sil], IV). Cela plus la connaissance de l'action de $\sigma$ sur $X$ (voir $\S 10.2$ ) nous permettent parfois de trouver le type de $X$ sans désingulariser $W$ jusqu'au bout. La fibre spéciale $W_{k}$ est intègre. Soit $e_{0}$ la spécialisation de l'élément neutre $o$ dans $W_{k}$, alors $\delta\left(e_{0}\right)=\lambda\left(e_{0}\right)=1$ car $\sigma(o)=o$. Donc $W_{k}$ admet au plus un point singulier $p_{0}$, et on a $\delta\left(p_{0}\right), \lambda\left(p_{0}\right) \leq 3$ (appliquer les lemmes 6 et 7). Pour la commodité du lecteur, nous suivons la numérotation de Tate [Tat] des différentes étapes de la désingularisation. La fin de chape étape est marquée par un signe.

(1) Supposons que $\nu\left(\Delta_{W}\right)=0$. Donc $W_{k}$ est lisse (ce qui équivaut à $\delta(p) \leq 1$ pour tout $\left.p \in W_{k}\right)$. Alors $X=W$ est de type $\mathrm{I}_{0}$.

(2) Supposons $\max \left\{\delta(p) \mid p \in W_{k}\right\}=2$. Alors $W_{k}$ est une courbe singulière semistable, et $X$ est de type $\mathrm{I}_{n}$, où $n=\nu\left(\Delta_{W}\right) \geq 1$.

Notons qu'inversement, si $X$ est de type $\mathrm{I}_{n}, n \geq 0$, alors $W_{k}$ est nécessairement semi-stable, donc $\delta(p) \leq 2$ pour tout point $p \in W_{k}$. Supposons à partir de maintenant que

$$
\exists p_{0} \in W_{k}, \quad \text { tel que } \delta\left(p_{0}\right)=3
$$

Il suit que $p_{0} \in W_{k}(k)$ (lemme 6 (d)-(e)).

(3) Supposons $\lambda\left(p_{0}\right)=1$, alors $W$ est régulier, donc $X=W$ est de type II.

Supposons à partir de maintenant que

$$
\lambda\left(p_{0}\right) \geq 2 \quad\left(\text { et } \delta\left(p_{0}\right)=3\right)
$$

Soit $Y_{1} \rightarrow W$ le morphisme construit dans $\S 6.3$. C'est aussi l'éclatement de $W$ de centre $p_{0}$. Comme $\lambda_{2}\left(p_{0}\right) \leq \delta\left(p_{0}\right)$, on a $\left[\lambda\left(p_{0}\right) / 2\right]=\left[\lambda_{2}\left(p_{0}\right) / 2\right]=1$. Donc $Y_{1}$ est régulier dans un voisinage du transformé strict de $W_{k}$ (lemme $\left.8(\mathrm{c})\right)$.

On note $W^{1}=W\left(p_{0}\right)$ le modèle de Weierstrass de $C$ associé à $p_{0}$ (voir déf. 12). Considérons le point

$$
p_{0}^{\prime} \in W^{1} \cap\left(W^{1} \wedge W\right)
$$

On a $\lambda\left(p_{0}^{\prime}\right)=2$ en vertu du lemme $9(\mathrm{~b})$.

(4-5) Supposons $\lambda\left(p_{0}\right)=2$, alors $X$ est de type III ou IV.

En effet, il suffit de montrer que $Y_{1}$ est régulier. Ce qui équivaut à $\lambda^{\prime}\left(p_{0}\right) \leq 1$. On a $\delta\left(p_{0}^{\prime}\right) \geq \lambda\left(p_{0}^{\prime}\right)=2$. Comme $W_{k}^{1}$ n'a pas de point double car $X$ serait de type $\mathrm{I}_{n}$, on a $\delta\left(p_{0}^{\prime}\right) \geq 3$. Donc $W_{k}^{1}$ est lisse en dehors de $p_{0}^{\prime}$ (lemme 6 (f)). D'où $\lambda^{\prime}\left(p_{0}\right) \leq 1$.

Notons que d'après le lemme 9 (d), l'image réciproque de $p_{0}$ dans $Y_{1}$ est une conique sur $k$. Il est aisé de voir ([Tat], §7) que cette conique est géométriquement irréductible (donc $X=Y_{1}$ est de type III) si et seulement si l'invariant $b_{8}$ vérifie $\nu\left(b_{8}\right)=2$. 
D'ici jusqu'à la fin de la section, on supposera que

$$
\delta\left(p_{0}\right)=\lambda\left(p_{0}\right)=3 .
$$

Comme $\lambda\left(p_{0}\right)$ est impair, d'après le lemme $9(\mathrm{~d})$ on a

$$
\sum_{p \in W_{k}^{1}, p \neq p_{0}^{\prime}} \delta(p)[k(p): k]=3 .
$$

(6-7) Supposons qu'il existe $q \in W_{k}^{1} \backslash\left\{p_{0}^{\prime}\right\}$ tel que $\delta(q)=1$. Alors $X$ est de type $\mathrm{I}_{n}^{*}, n \geq 0$.

En effet, on a $\lambda(q)=2$, et donc l'image réciproque de $q$ dans la désingularisation minimale de $W^{1}$ est une composante $\simeq \mathbb{P}_{k}^{1}$ de multiplicité 1 (voir 9.3.1). D'autre part, $W^{1}$ a au moins trois points singuliers, donc $X$ domine $Y_{1}^{\prime}$ (on peut aussi utiliser le corollaire 5). Il suit que $X$ est de type $\mathrm{I}_{n}^{*}$ en considérant la liste de toutes les configurations possibles de $X_{k}$. Si $\delta(p) \leq 1$ pour tout $p \neq p_{0}^{\prime}$, alors $n=0$. Sinon, il existe un unique point $p_{1} \in W_{k}^{1}(k)$ tel que $\delta\left(p_{1}\right)=2$ d'après l'égalité $(* *)$ ci-dessus. L'algorithme décrit dans 9.3.3 permet de déterminer l'entier $n$.

Il reste le cas où il existe $p_{1} \in W_{k}^{1} \backslash\left\{p_{0}^{\prime}\right\}$ avec $\delta\left(p_{1}\right)=3$. Alors $W^{1}$ n'a que deux points singuliers $p_{0}^{\prime}$ et $p_{1}$. Il suit que $X$ est de type II*, III* ou IV*.

(8) Supposons $\lambda\left(p_{1}\right)=2$, alors $X$ est de type $\mathrm{IV}^{*}$. En effet, on a $\lambda^{\prime}\left(p_{1}\right)=1$. Donc $C$ admet exactement trois modèles de Weierstrass minimaux qui sont $W, W^{1}$ et $W^{2}:=W^{1}\left(p_{1}\right)$, et $W_{k}, W_{k}^{2}$ sont réduits. On conclut grâce à $\S 10.2$.

(9) Supposons $\lambda\left(p_{1}\right)=3$ et $\lambda^{\prime}\left(p_{1}\right) \geq 2$, alors $X$ est de type III*. En effet, $C$ admet alors au moins quatre modèles de Weierstrass minimaux.

(10) Supposons $\lambda\left(p_{1}\right)=3$ et $\lambda^{\prime}\left(p_{1}\right)=1$, alors $X$ est de type II*. Cela se voit comme dans (8) ci-dessus en notant que les modèles de Weierstrass minimaux de $C$ sont $W, W^{1}$ et $W^{2}:=W^{1}\left(p_{1}\right)$ et que $W_{k}^{2}$ n'est pas réduit.

Remarque 22. La désingularisation de $p_{0}$ décrite dans cette section s'applique plus généralement à un point singulier $p_{0}$ d'un modèle de Weierstrass $W$ d'une courbe hyperelliptique de genre $\geq 1$, tel que $\delta\left(p_{0}\right), \lambda\left(p_{0}\right), \lambda^{\prime}\left(p_{0}\right) \leq 3$. En effet la désingularisation d'un point est une question locale.

\section{RÉFÉRENCES}

[Art] M. Artin, Lipman's proof of resolution of singularities for surfaces, Arithmetic geometry (Cornell and Silverman, eds.), Springer-Verlag, 1986, pp. 267-287. MR 86:1980

[Chi] T. Chinburg, Minimal models for curves over Dedekind rings, Arithmetic geometry (Cornell and Silverman, eds.), Springer-Verlag, 1986, pp. 309-326. MR 86:1982

[Des] M. Deschamps, Réduction semi-stable, Séminaire sur les pinceaux de courbes de genre au moins deux, Astérisque, vol. 86, 1981, pp. 1-34.

[Har] R. Hartshorne, Algebraic Geometry, Graduate Texts in Math., 52, Springer-Verlag, 1977. MR 57:3116

[Kau] I. Kausz, Eine Abschätzung der Selbstschnittzahl des kanonischen Divisors auf arithmetischen Flächen mit hyperelliptischer generischer Faser, Dissertation, Köln (1995).

[Kod] K. Kodaira, On compact analytic surfaces, II, Ann. of Math. 77 (1963), 563-626. MR 89m:11059

[Lan] S. Lang, Introduction to Arakelov Theory, Springer-Verlag, 1988.

[Lic1] S. Lichtenbaum, Duality theorems for curves over p-adic fields, Invent. Math. 7 (1969), 120-136. MR 39:4158

[Lic2] S. Lichtenbaum, Curves over discrete valuation rings, Amer. J. Math. 90 (1968), 380-405. MR 37:6284

[Lip] J. Lipman, Rational singularities, Publ. Math. 36 (1969), 195-279. MR 43:1986

[Liu1] Q. Liu, Modèles minimaux des courbes de genre deux,, J. Reine Angew. Math. 453 (1994), 137-164. MR 95k:14024 
[Liu2] Q. Liu, Conducteur et discriminant minimal de courbes de genre 2, Compositio Math. 94 (1994), 51-79. MR 96b:14038

[Loc] P. Lockhart, On the discriminant of hyperelliptic curve, Trans. Amer. Math.Soc. 342 (1994), 729-752. MR 94f:11054

[Mat] H. Matsumura, Commutative Algebra, second edition, Benjamin/Cummings, New York, 1980. MR 82i: 13003

[Mil1] J. S. Milne, Jacobian varieties, Arithmetic geometry (Cornell \& Silverman,eds.), SpringerVerlag, 1986, pp. 167-212. MR 86:1976

[Mil2] J. S. Milne, Étale cohomology, Princeton Univ. Press, Princeton, New Jersey, 1980. MR 81j:14002

[N-U] Y. Namikawa, K. Ueno, The complete classification of fibers in pencils of curves of genus two, Manuscripta Math. 9 (1973), 143-186. MR 51:5595

[Nér] A. Néron, Modèles minimaux de variétés abélienne, Publ. Math. IHES 21 (1964). MR 31:3423

[Ogg] A. P. Ogg, On pencils of curves of genus two, Topology 5 (1966), 355-362. MR 34:3423

[Sai] T. Saito, Conductor, discriminant, and the Noether formula of arithmetic surfaces, Duke Math. Jour. 57 (1988), 151-173. MR 89f:14024

[Sil] J. Silverman, Advanced Topics in the Arithmetic of Elliptic Curves, Graduate Texts in Math., 151, Springer-Verlag, 1994. MR 96b:11074

[Tat] J. Tate, Algorithm for determining the type of a singular fiber in an elliptic pencil, Lect. Notes in Math., vol. 476, Springer Verlag, 1975, pp. 33-52. MR 52:13850

[Uen] K. Ueno, Discriminants of curves of genus 2 and arithmetic surfaces, Algebraic geometry and commutative algebra, in honor of Masayaoshi Nagata, vol. II (1987), 749-770. MR 90a: 14040

[Vie] E. Viehweg, Invarianten der degenerierten Fasern in lokalen Familien von Kurven, J. Reine Angew. Math. 293 (1977), 284-308. MR 16655

CNRS, Laboratoire de Mathématiques Pures, Université Bordeaux I, 351, Cours de la libération, 33405 Talence Cedex, France

E-mail address: liu@math.u-bordeaux.fr 